\title{
On the heat transfer effects of nanofluids within rotor-stator cavities
}

Cite as: Phys. Fluids 30, 082007 (2018); https://doi.org/10.1063/1.5043264

Submitted: 07 June 2018 . Accepted: 11 August 2018 . Published Online: 29 August 2018

D. Fernando, S. Gao, and S. J. Garrett (iD)

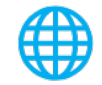

\section{ARTICLES YOU MAY BE INTERESTED IN}

Vibrational modes prediction for water-air bubbles trapped in circular microcavities

Physics of Fluids 30, 082001 (2018); https://doi.org/10.1063/1.5037328

Fluid flow and heat transfer over a rotating and vertically moving disk

Physics of Fluids 30, 063605 (2018); https://doi.org/10.1063/1.5037460

The effect of surface roughness on rotor-stator cavity flows

Physics of Fluids 30, 064103 (2018); https://doi.org/10.1063/1.5028209

PHYSICS TODAY

WHITEPAPERS
ADVANCED LIGHT CURE ADHESIVES

Take a closer look at what these

environmentally friendly adhesive

systems can do
READ NOW

PRESENTED BY 8. MASTERBOND* 


\title{
On the heat transfer effects of nanofluids within rotor-stator cavities
}

\author{
D. Fernando, ${ }^{\text {a) }}$ S. Gao, ${ }^{\text {b) }}$ and S. J. Garrett ${ }^{\mathrm{c})}$ \\ Department of Engineering, University of Leicester, Leicester LE1 7RH, United Kingdom
}

(Received 7 June 2018; accepted 11 August 2018; published online 29 August 2018)

\begin{abstract}
Owing to the rapid development of a number of technological and industrial sectors, high-performance electronic devices are now ubiquitous in modern engineering and industrial applications. Effective heat management is crucial to the smooth operation of such devices, and sometimes conventional methods of heat transfer fail to deliver the required performance. Recent advances in the field of nanofluids are a promising route to improve heat-transfer performance, and this is our motivation. We propose two computational fluid dynamics models for a rotor-stator cavity operating at $\operatorname{Re}_{\omega}=1.0 \times 10^{5}$ and filled with a fluid that consists of different volume fractions of $\mathrm{Al}_{2} \mathrm{O}_{3}$ nanoparticles. The first model simulates the nanofluid mixture using a single-phase transport model, and the second approach uses a two-phase transport model that allows for the relative velocity between the particle and fluid phases. All simulations are conducted using the second-order accurate solver, OpenFOAM ${ }^{\circledR}$, that is based on the finite volume method and using Large eddy simulation methods. Our results show that the higher volume fractions of $\mathrm{Al}_{2} \mathrm{O}_{3}$ nanoparticles can achieve higher heat transfer rates, and at the same time, dilute nanoparticle concentrations have subtle effects on the momentum transport of the system. This is an advantage over micro-particle dispersion. Furthermore, we consider the effects of particle forces in the two-phase model, such as Brownian and thermophoresis forces, and suggest that the thermophoresis forces are the dominant effect within the cavity geometry. Published by AIP Publishing. https://doi.org/10.1063/1.5043264
\end{abstract}

\section{INTRODUCTION}

The non-isothermal fluid flow over a rotating disk has been investigated thoroughly in the previous literature. ${ }^{1-5}$ As a result, we now know that the induced fluid motion due to the disk's surface rotation is a critical driving force of the heat transfer properties of the resulting boundary-layer flow. The physics behind these aspects is therefore essential to understand and improve the heat transfer rates in many practical applications, such as rotating machinery, combustion chambers, and hot-fluid mixtures.

Early investigations of the non-isothermal flow date back to 1950s and followed closely progress being made on isothermal rotating boundary layers. By assuming the similarity solutions of von Kármán, ${ }^{6}$ Millsaps ${ }^{7}$ investigated the laminar heat transfer coefficients (or the Nusselt numbers) of the rotating disk. Later, Cobb and Saunders ${ }^{8}$ extended this to turbulent flows by conducting a series of experimental investigations. It was found that the turbulent flow significantly increases the heat transfer coefficients of rotating-disk flows.

Nikitenko ${ }^{9}$ conducted the first experimental investigations on heat transfer within rotor-stator cavities. He investigated a range of cavities with various aspect ratios $0.018<G$ $=h / r<0.085$ operating close to $\operatorname{Re}_{\omega}=1 \times 10^{6}$ with the aim of obtaining generalised correlations for local Nusselt numbers, $N u_{r}=h_{T} r / k_{T}$, for both laminar and turbulent flows. Here $h_{T}$ and $k$ are the heat transfer coefficient and the thermal conductivity of the fluid, respectively. The resulting

a)bdrf1@leicester.ac.uk

b)sg32@leicester.ac.uk

c) Author to whom correspondence should be addressed: sjg50@ leicester.ac.uk local Nusselt number correlations on the rotor were found to take the form $N u_{r}=0.675 \mathrm{Re}_{\omega, \text { local }}^{0.5}$ for laminar flow and $N u_{r}=0.02175 \mathrm{Re}_{\omega . \text { local }}^{0.8}$ for the turbulent flow. Crucially both were independent of the cavity aspect ratio.

Some years later, Owen et al. ${ }^{10}$ conducted a combined theoretical and experimental investigation on the heat transfer within various rotating-disk configurations operating within the turbulent regime. These include the free disk (equivalent to the von Kármán case), rotor-stator cavity, and rotor-stator cavity with through flow. In each configuration, the rotor was assumed to be the fixed heat source. For the rotor-stator cases, various aspect ratios between $G=0.0067-0.18$ were tested at $2 \times 10^{5} \leq \operatorname{Re}_{\omega} \leq 4 \times 10^{6}$. The results showed that the (spatially) mean Nusselt number of the free disk followed $\overline{N u_{r, d}}=0.017 R e_{\omega}^{0.814}$. For cavities, the mean Nusselt number was found to be independent of the aspect ratio only for large aspect ratios where $G \geq 0.06$. For very small aspect ratios, $G<0.01$, the flow was found to be of Couette type (i.e., 'merged boundary layers' in the words of Daily and Nece ${ }^{11}$ ) and $\overline{N u_{r}}>\overline{N u_{r, d}}$. For moderate aspect ratios, $0.01 \leq G \leq 0.06$, the rotation of the core region of the cavity was observed to be of the Batchelor type (i.e., unmerged boundary layers), and $\overline{N u_{r}}<\overline{N u_{r, d}}$. These results imply that, as the aspect ratio is increased from small values, the mean Nusselt number first decreases to a minimum value at $G \approx 0.01$, then increases. For about $G>0.06$, the heat transfer effects from the stator are diminished and the flow resembles that of a single disk with $\overline{N u_{r}} \approx \overline{N u_{r, d}}$. In addition, their studies showed that non-zero through-flow has a great influence on heat transfer rates, and average Nusselt numbers tend to increase at a higher rate of through flow. 
These early stage investigations concentrated on clarifying the heat-transfer properties of the flow under laminar vs. turbulent conditions and also the geometrical dependency of the rotor-stator systems. Most work completed prior to the 1990s was focussed in these directions and was presented with this in mind. Owen and Rogers ${ }^{12,13}$ did an excellent job of summarising the advances of this period. During the 1990s, general advancements in our understanding of the rotating disk boundary layers ${ }^{14-19}$ were a significant influence on heat transfer studies in both single- and two-disk systems. Our attention will now move to more recent experimental and numerical investigations into the heat transfer of cavity systems.

Elkins and Eaton ${ }^{20}$ conducted a detailed experimental investigation of heated rotating disks operated at $\operatorname{Re}_{\omega}=1$ $\times 10^{6}$, which is in the turbulent regime. Due to the lowtemperature difference maintained during the investigation, the buoyancy effects are subtle, and the temperature distributions have only a minor effect on the velocity fields of the flow. It was found that the heat transfer coefficient was fairly constant in the laminar flow region, but varies as $r^{7}$ and $r^{0.6}$ in the transitional and turbulent regions, respectively. At inner regions $(<z / \delta \approx 3)$, the structure parameter $a_{1}=\left(\overline{u_{r}^{\prime} u_{\theta}^{\prime}}+\overline{u_{\theta}^{\prime} u_{z}^{\prime}}\right)^{1 / 2} / 2 k$ and correlation coefficients $R_{u \theta}$ and $R_{v \theta}$ are reduced when compared to the corresponding values in two-dimensional boundary layers. This suggests that the three-dimensionality of the boundary layer affects both turbulent shear stresses and heat flux. The outer region of the boundary layer, $z / \delta>3$, shows a similar trend as the inner region for the parameters $a_{1}$ and $R_{u \theta}$, but the variation of $R_{v \theta}$ is similar to two-dimensional boundary cases. The values of turbulent Prandtl numbers, $P r_{t}$, are slightly reduced compared to the two-dimensional boundary layers ${ }^{21,22}$ in the either side of the three-dimensional boundary layer. A further explanation has been developed using a quadrant analysis of the boundary layer, ${ }^{23}$ and this revealed that there is a significant deficit in sweep contributions on turbulent shear stresses, whereas both ejections and sweeps contribute to the vertical turbulent heat flux.

The first significant numerical investigation on heat transfer on the rotor-stator cavity was conducted by Serre et al. ${ }^{1}$ and used direct numerical simulation (DNS) for a rotor-stator cavity with aspect ratio $G=0.426$ operated at $R e_{\omega}=1 \times 10^{5}$. The study visualised the instantaneous temperature distributions inside the cavity and noted extruded large-scale hot structures toward the upper rotor layer, which is a result of Ekman pumping. A region of hot flow was also found to spread from the rotor hub to one-third of the effective radius of the cavity, $\Delta R=r_{2}-r_{2}$, where $r_{2}$ and $r_{1}$ are the outer and the inner radii of the annular cavity, respectively. The buoyancy effects on the flow were tested using Rayleigh numbers $R a=0,2 \times 10^{4}$, and $2 \times 10^{6}$ and, as described by Elkins and Eaton, were found to be less sensitive to mean velocity fields, but more pronounced in the mean temperature fields. In the two higher Rayleigh number cases, a significant decrease in temperature was noticed near the rotor and stator boundary layers but, in both cases, the temperature distributions of the core region were reasonably similar to the zero Rayleigh number case. Later, Poncet and Serre ${ }^{2}$ conducted a similar investigation with a cavity of $G=0.2$ over a range of Reynolds and Rayleigh numbers up to $\operatorname{Re}_{\omega}=1 \times 10^{6}$ and $R a=1 \times 10^{8}$ by which point both the rotor and stator boundary layers are turbulent. This latter paper used a novel large eddy simulation (LES) approach suitable for spectral methods and, having found results consistent with the earlier paper, is important more from the numerical perspective.

In 2009, Tuliszka-Sznitko et l. $^{3}$ investigated the heat transfer properties of rotor-stator cavities using LES methods. They used cavities in two different configurations, $G=0.2$, $\operatorname{Re}_{\omega}=3 \times 10^{5}$ and $G=0.11, \operatorname{Re}_{\omega}=1.5 \times 10^{5}$, and the thermal Rossby numbers of the simulations were selected to be between 0.01 and 0.4 . In each case, they obtained the local Nusselt number distribution over the rotor and stator. They observed that an increase in Re results in an increased local Nusselt number, which implies a higher heat transfer rate. The influence of thermal Rossby number on the local Nusselt numbers was shown to be minimal. These simulations were extended in 2010 to co-and counter-rotating cavities. ${ }^{4}$

Two years later, Tuliszka-Sznitko et al. ${ }^{5}$ conducted rotorstator simulations using DNS and LES approaches for the range of aspect ratios and Reynolds numbers, $G=0.02-0.5$, $\operatorname{Re}_{\omega}=1 \times 10^{5}-2.9 \times 10^{5}$. The axial distributions of Reynolds stress components, temperature fluctuations, and turbulent heat fluxes were validated with previous experiments results of Elkins and Eaton. ${ }^{20}$ The structural parameters were found to be less sensitive to the Reynolds stress components and turbulent heat fluxes than the changes in geometric parameters, such as $G$ and the radius of curvature $R_{m}=\left(r_{2}+r_{1}\right) /\left(r_{2}-r_{1}\right)$ for annular cavities.

Recently, Turkyilmazoglu ${ }^{24}$ used the von Kármán transformation to obtain the exact solutions for co-axially mounted stretchable disks that rotate in co- and counter-rotating directions. The primary motivation of the study was to explain the momentum transport and heat transfer properties under the stretching mechanism of the disks. It was found that the stretching mechanism has a significant effect on the main flow behaviors. Furthermore, it was observed that the stretching of the upper disk increases the heat transfer at all rotation rates.

Many investigations of the heat transfer of non-isothermal disks have focused on identifying and understanding the convective heat transfer patterns of rotor-stator systems, which may eventually help to improve their heat-transfer capabilities. However, the weak thermal conductivities of common working fluids such as air and water impose an unavoidable bottleneck on performance that prevents such systems from being used in high-performance applications areas. Maxwell ${ }^{25}$ proposed a remedy to this problem by mixing micro-sized metallic and metal oxide particles into the working fluid. Even though the thermal conductivities are improved as expected, many practical applications of micro-particle dispersions have suffered undesirable side effects (see Ref. 26). It seems that the micro-scale particles are too heavy for the streamlined operation of many engineering and industrial devices. However, the emergence of nanotechnology now allows the mass production of nanoscale particles, which are much more resistant to the drawbacks experienced in micro-particle dispersions. Choi and Eastman ${ }^{27}$ proposed nanoparticle suspensions for enhanced heat transfer applications and the resulting particle-fluid mixture is now typically called a nanofluid. 
The work on nanofluids has progressed rapidly over the last couple of decades and much of the previous work ${ }^{28-31}$ has been based on the study of the potential heat-transfer enhancement of nanofluids within different application areas. Their use has been found to be very successful in practice. Alinia et al..$^{32}$ and Goodarzi et al. ${ }^{33}$ used a two-phase mixture model to study the effects of the nanoparticle slip-velocity that results in inhomogeneous volume faction distributions across the computational domain. Ghasemi and Aminossadati ${ }^{34}$ studied the effects of Brownian motion on the nanoparticles in a triangular cavity using the Koo-Kleinstreuer thermo-physical model, ${ }^{35}$ and observed improvements in heat transfer rates due to Brownian slip motion.

Later, a series of investigations emerged that studied nanoparticle slip mechanisms, as described by Buongiorno. ${ }^{36}$ Mahajan and Sharma ${ }^{37}$ used a theoretical model based on the Brownian, thermophoresis, and magnetophoresis slip mechanisms to study the penetrative convection in a thin magnetic nanofluid layer under an applied magnetic field and an internal heat source. Linear stability theory was used to predict the onset of the penetrative convection under the important intrinsic and extrinsic parameters of the magnetic nanofluid. Avramenko et al $^{38}$ studied the Hiemenz flow of a nanofluid by considering the self-similar form of the momentum, energy, and concentration equations. The functional dependencies of the thermophysical properties of nanofluids were included by considering the concentration and temperature profiles of the fluid in which the slip velocities between the particles and fluid are included. This was done by considering the Brownian diffusion and the thermophoresis effects. Hayat et al. ${ }^{39}$ and Ramzan et al. $^{40}$ investigated the second-order nanofluid flow past a bidirectional stretched surface using Cattaneo-Christov double diffusion, and the latter study incorporated the variable thermal conductivities on magnetohydrodynamic flows. In both cases, the Brownian diffusion and thermophoresis effects were retained in their models.

Surprisingly, nanofluid heat transfer has not been widely studied in the rotor-stator flow domain. However, there are a few investigations that concern the potential nanofluid heat transfer enhancement in single rotating-disk boundary layers. In particular, Bachok et al. ${ }^{41}$ studied the nanofluid heat transfer over a rotating disk with surface mass flux (used as a proxy for a porous surface) and conducted a numerical simulation by solving the transformed boundary-layer equations using a finite difference method. Here the nanofluid consists of nanoparticles of $\mathrm{Al}_{2} \mathrm{O}_{3}, \mathrm{CuO}$, and $\mathrm{TiO}_{2}$ suspended in water. The mixture's thermal conductivities were calculated based on Maxwell's model ${ }^{25}$ and a model developed by Patel et al. ${ }^{42}$ As the nanoparticle concentration increases up to $20 \mathrm{vol} . \%$, the latter model predicts increased heat transfer rates on both suction and injection at the rotor surface, whereas the former model only predicts increased heat transfer rates under injections. More recently, Turkyilmazoglu ${ }^{43}$ studied the flow and heat transfer rates of a rotating disk with these same nanofluid suspensions in water. That study was predominately concerned with the variations to the momentum boundary layers under increased nanoparticle concentrations. The resulting boundary-layer velocity profiles suggest that an increase in concentrations of certain nanoparticles, such as $\mathrm{Al}_{2} \mathrm{O}_{3}$ or $\mathrm{TiO}_{2}$ increase the amount of fluid entrained into the rotor boundary layer, but nanoparticles of $\mathrm{CuO}$ and $\mathrm{Cu}$ behave oppositely. Regardless of the type of the nanoparticle, the increase in particle concentration resulted in higher local Nusselt numbers, which is an indication of enhanced heat transfer rates. Later, Mustafa et al. ${ }^{44}$ investigated a Bödewadt boundary layer over a stationary stretching disk. More recently, Mushtaq and Mustafa ${ }^{45}$ studied a nanofluid flow over a stretching rotating disk with an axial magnetic field using the Buongirno model for the Brownian diffusion and thermophoresis forces.

These prior investigations on rotating disk boundary layers are based on the theoretical solutions of the von Kármán similarity velocity profiles which, while accurate, require underlying assumptions that are too restrictive compared to full-fledged numerical methods. Furthermore, the flow fields are assumed to be fully laminar, and no indication of the turbulent behavior of the flow has been given, nor has consideration been given to particle diffusion due to Brownian and thermophoresis effects.

In this current study, we propose finite volume method (FVM)-based LES computational models for the rotor-stator flow. An operational Reynolds number of $\operatorname{Re}_{\omega}=1 \times 10^{5}$ will be used in our simulations. The previous LES of Séverac et al. ${ }^{46}$ and Makino et al. ${ }^{47}$ remarked that $R e_{\omega}=1 \times 10^{5}$ is high enough to generate a turbulent stator but will leave a considerable portion of the rotor boundary layer within the laminar regime. First, a single-phase $S_{P}$ transport model is used to demonstrate the effects of nanofluids on the momentum and thermal distributions within the rotor-stator cavity. Subsequently, a two-phase model, $T_{P}$, based on the Buongirno model is used to understand the dynamics and behavior of the nanoparticle phase inside the cavity. Although we consider only $\mathrm{Al}_{2} \mathrm{O}_{3}$ nanoparticles here, a popular variant in the literature, our method is readily generalised to other nanoparticle species. Note that the volume fraction is defined in an obvious way,

$$
\phi=\frac{\text { Volume of } \mathrm{Al}_{2} \mathrm{O}_{3} \text { constituent }}{\text { Volume of all constituents }} .
$$

The mean velocity profiles and the turbulence intensity profiles of both $S_{P}$ and $T_{P}$ simulation models at a very low volume fraction will be validated against the results of the previous conventional-fluid investigation due to Séverac et al. ${ }^{46}$ The momentum and thermal properties of the nanofluid with different nanoparticle volume fractions, $\phi=0.02,0.04$, and 0.2, will then be evaluated for the $S_{P}$ model and compared to clarify the advantages of nanofluids over the conventional fluids within rotor-stator flows.

In Sec. III C, the outcomes will be discussed for a similar set of simulations for the $T_{P}$ simulation model. Finally, the local and the mean Nusselt number distributions will be presented to show the quantitative increases in the heat transfer performance of the rotor-stator cavities filled with $\mathrm{Al}_{2} \mathrm{O}_{3}$ based nanofluid.

\section{COMPUTATIONAL MODELS}

\section{A. Geometrical and numerical modeling}

Rotor-stator cavities can be considered to contain an Ekman $^{48}$ boundary layer over the rotor and a Bödewadt ${ }^{49}$ 
boundary layer over the stator. In our geometrical model, the inter-disk spacing, $h$, between the two disks is large enough to ensure that these boundary layers remain distinct and do not merge. We consider an annular cavity with inner radius, $r_{1}$, determined by the radius of the hub directly connected to the rotating disk. The distance between the origin and the periphery of the rotating disk defines the outer radius, $r_{2}$, at which point a wall is fixed to the stator. The effective radial extent of the annular cavity is therefore $\Delta R=r_{2}-r_{1}$. Figure 1(a) illustrates a typical side view of the rotor-stator cavity. The aspect ratio, $G$, and the radius of curvature, $R_{m}$, are important geometrical parameters of the cavity and are defined as

$$
G=\frac{h}{r_{2}-r_{1}}=0.2, \quad R_{m}=\frac{r_{2}+r_{1}}{r_{2}-r_{1}}=1.8 .
$$

The operational Reynolds number is based on the rotating-disk periphery and is taken to be $\operatorname{Re}_{\omega}=\omega r_{2}^{2} / v=1 \times 10^{5}$. The local Reynolds number at any given point $p$ on the disk surface is $\operatorname{Re}_{\text {local }, \omega}=\omega r^{2} / v$, where $r$ is the radial distance between the $p$ and the origin. The clearance gaps $\delta_{1}$ and $\delta_{2}$, as illustrated in Fig. 1(a), are important to separate rotating and stationary components. These gaps are smaller than the threshold values specified in Séverac et $a l .{ }^{46}$ and so are expected to lead to negligible effects on the main flow field. Séverac et al. used an exponentially decaying boundary function for the tangential velocity component to mimic the effects of these gaps. In our case, the gaps are implemented at the geometrical level.

The commercial mesh generation software ANSYS ICEM $\mathrm{CFD}{ }^{\circledR}$ is used to generate a hexahedron computational mesh with multiple O-grids (see Ref. 50). Figures 1(b) and 1(c) illustrate the side and top views of the mesh that contains over $5 \times 10^{6}$ cells $(r \times \theta \times z=220 \times 180 \times 140)$. A justification of the mesh resolution for the current simulation models will be given in Sec. III.

All simulations are conducted using OpenFOAM ${ }^{\circledR}$, and a second-order accurate collocated FVM based segregate solver $^{51}$ is used for solving the governing equations. A second-order accurate central difference schema and implicit backward difference method are used to determine the convective and temporal terms, respectively. The pressure-velocity coupling is achieved using the non-iterative Pressure-Implicit with Splitting of Operators (PISO) algorithm. ${ }^{52}$ The current study relies on LES methods to evaluate turbulent stresses on the flow field. The non-linear convective term of the filtered momentum equations results in sub-grid scale stresses, $\tau_{\text {sgs }}$, which can be described by Eq. (2). Our simulation uses a walladapting local eddy-viscosity (WALE) ${ }^{53}$ sub-grid scale model to calculate the sub-grid scale viscosity, $\mu_{s g s}$, associated with $\tau_{s g s}$, and Eq. (3) describes the relevant expression,

$$
\begin{gathered}
\tau_{s g s}-\frac{1}{3} \operatorname{tr}\left(\tau_{s g s}\right)=-2 \mu_{s g s} \overline{\boldsymbol{S}}, \\
\mu_{s g s}=\rho \overline{\Delta_{s}} \frac{\left(\boldsymbol{S}^{d} \boldsymbol{S}^{\boldsymbol{d}}\right)^{3 / 2}}{(\overline{\boldsymbol{S}} \overline{\boldsymbol{S}})^{5 / 2}+\left(\boldsymbol{S}^{\boldsymbol{d}} \boldsymbol{S}^{\boldsymbol{d}}\right)^{5 / 4}} .
\end{gathered}
$$

Here $\boldsymbol{S}=\frac{1}{2}\left(\nabla \boldsymbol{u}+\nabla \boldsymbol{u}^{T}\right)$ is the strain rate tensor, and $\overline{\boldsymbol{S}}$ and $S^{d}$ are the filtered and deviatoric parts of $S$, respectively, $\operatorname{tr}(\cdot)$ is the trace of a tensor, and $\rho$ is the density of the fluid. The quantity $\Delta_{s}=C_{w} V^{1 / 3}$ is the filter width of the WALE model parametrised by a constant, $C_{w}=0.325$, and the volume of cells $(V=\sqrt{\Delta x \Delta y \Delta z})$. The turbulent heat fluxes are determined by assuming the sub-grid scale Prandtl number of $P r_{s g s}=0.4$ (see Ref. 54). The near-wall mesh density is increased to resolve the laminar sublayer on the boundary layers, and the dimensionless wall-normal distance to the cell centres of the closest cell elements to the walls are usually maintained well below unity. That is, we impose relevant $z_{\max }^{+}, r_{\max }^{+} \leq 1.0$ conditions at the disk surfaces, the rotor hub, and the outer wall of the cavity. The time steps $\Delta t$ have been chosen to satisfy the Courant number conditions of $C_{\max }=\Delta t \sum_{i=1}^{n} \frac{u_{i}}{\Delta x_{i}} \approx 0.3$, where $i=3$ for this three-dimensional case.

\section{B. Single-phase model}

The single-phase transport model assumes that the multiple phases of the flow are in thermal equilibrium and possess

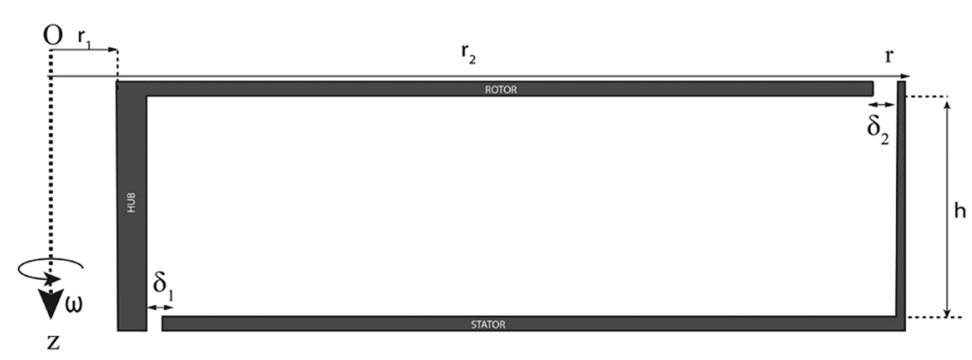

(a)

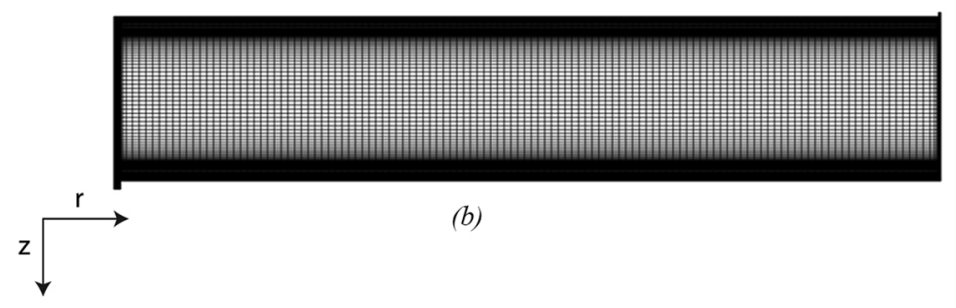

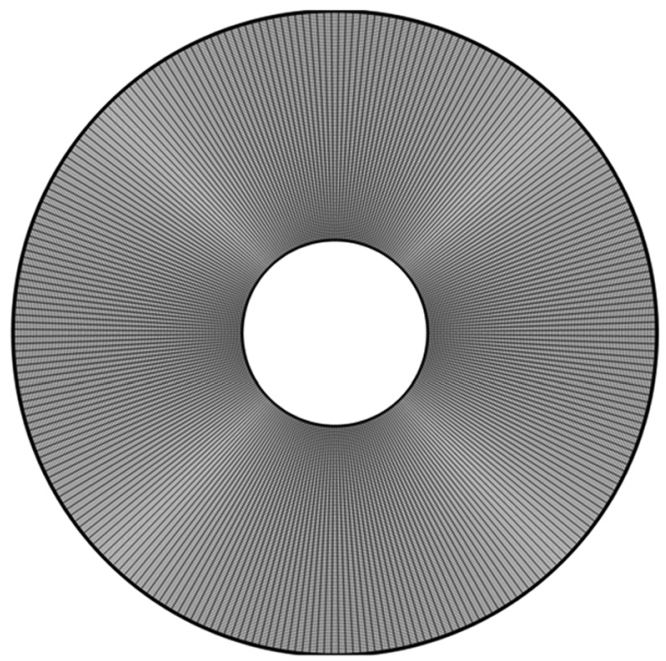

(c)

FIG. 1. The geometrical and mesh configurations, (a) side view of rotor-stator cavity, (b) side, and (c) top views of the current computational mesh, $R e_{\omega}=1 \times 10^{5}$. 
zero relative velocity. This avoids a dedicated governing equation for particle phase volume fractions. Furthermore, the particle phase is assumed to be evenly spread over the primary phase (water), resulting in a nanofluid mixture. The thermo-physical properties of the nanofluid mixture can be calculated from correlation models (described in Sec. II D) and fed into the incompressible Navier-Stokes equations appropriately modified for nanofluids,

$$
\begin{gathered}
\frac{\partial \rho_{n f}}{\partial t}+\nabla \cdot \rho_{n f} \boldsymbol{u}=0, \\
\frac{\partial \rho_{n f} \boldsymbol{u}}{\partial t}+\nabla \cdot \rho_{n f} \boldsymbol{u} \boldsymbol{u}=-\nabla P+\nabla \cdot \tau_{e f f}+\rho_{n f} \boldsymbol{g}_{\boldsymbol{b}}, \\
(\rho c)_{n f}\left[\frac{\partial T}{\partial t}+\boldsymbol{u} \cdot \nabla . T\right]=\nabla .\left(k_{e f f} \nabla T\right) .
\end{gathered}
$$

Here $\boldsymbol{u}=\left(u_{x}, u_{y}, u_{z}\right)$ is the velocity of the mixture, and the vector operator $\nabla$ is implicitly defined for Cartesian coordinates. The quantities $\rho$ and $c$ are the density and heat capacity at constant pressure, and the subscripts $(\cdot)_{n f}$ represent the equivalent nanofluid properties resulting from the correlation models of Sec. II D. $\boldsymbol{g}_{\boldsymbol{b}}=\left[1-\beta_{n f}\left(T-T_{r e f}\right)\right] \boldsymbol{g}$ is the Boussinesq gravity evaluated using the Boussinesq approximation. ${ }^{55}$ Here $\beta_{n f}, T, T_{\text {ref }}$, and $g=9.81 \mathrm{~m} \mathrm{~s}^{-2}$ are thermal expansion coefficient of the nanofluid mixture, temperature, reference temperature, and gravitational acceleration, respectively.

Since the volume fractions of nanoparticles are homogeneous throughout the domain, the single-phase models resemble the standard incompressible Navier-Stokes equations, but the simulations are tightly coupled with a given volume fraction. Furthermore, the effective viscosities and thermal conductivities of the above equations can be calculated using $\mu_{s g s}$ and $\operatorname{Pr}_{\text {sgs }}$ within

$$
\begin{gathered}
\mu_{e f f}=\mu_{n f}+\mu_{s g s}, \\
k_{e f f}=k_{n f}+(\rho c)_{n f} \frac{\mu_{s g s}}{P r_{s g s}} .
\end{gathered}
$$

\section{Two-phase model}

The two-phase transport models allow for a slip velocity (i.e., relative velocity) between two phases of fluid, and here we opt to use a mixture model ${ }^{56}$ to implement this. Such models are particularly suitable for a multiphase flow with non-zero slip velocity between each phase.

In the traditional mixture model, the slip velocity is considered to arise from an imbalance in the local forces of nanoparticles and is presented as a function of particle relaxation time, particle drag forces, and advection due to the local mixture velocity. ${ }^{57}$ Our investigation, however, adopts an alternative approach that calculates slip velocities as described by Buongiorno. ${ }^{36}$ In particular, Buongiorno suggests that inertia, Brownian diffusion, thermophoresis, diffusiophoresis, Magnus effects, fluid drainage, and gravity are all important, with Brownian diffusion and thermophoresis the dominant effects in laminar flows. The presence of turbulence can suppress the Brownian and thermophoresis effects, but these effects should still play a significant role in near-wall (i.e., boundarylayer) regions. By considering the Brownian and thermophoresis effects, the governing equations for a nanofluid can be rewritten as follows:

$$
\begin{gathered}
\frac{\partial \rho_{n f}}{\partial t}+\nabla \cdot \rho_{n f} \boldsymbol{u}=0, \\
\frac{\partial \rho_{n f} \boldsymbol{u}}{\partial t}+\nabla \cdot \rho_{n f} \boldsymbol{u} \boldsymbol{u}=-\nabla P+\nabla \cdot \tau_{e f f}+\rho_{n f} \boldsymbol{g}_{\boldsymbol{b}}, \\
(\rho c)_{n f}\left[\frac{\partial T}{\partial t}+\boldsymbol{u} \cdot \nabla \cdot T\right] \\
=\nabla \cdot\left(k_{e f f} \nabla T\right)+\left(\rho_{p} c_{p}\right)\left[D_{B} \nabla \phi \cdot \nabla T+D_{T} \frac{\nabla T \cdot \nabla T}{T}\right], \\
{\left[\frac{\partial \phi}{\partial t}+\boldsymbol{u} \cdot \nabla \cdot \phi\right]=\nabla \cdot\left[\left(D_{B}+\epsilon_{P}\right) \nabla \phi+D_{T} \frac{\nabla T}{T}\right] .}
\end{gathered}
$$

Here $\phi$ is the volume fraction and $D_{B}, D_{T}$ are the Brownian and thermophoresis diffusion coefficients, respectively. In addition to assuming an incompressible fluid, Buongiorno considers the following assumptions necessary for a nanofluid mixture and, where appropriate, each applies to both the base fluid and the nanoparticles:

1. No chemical reactions.

2. Negligible external forces.

3. The mixture is diluted, $\phi \ll 1$.

4. Viscous dissipations are negligible.

5. Radiative heat transfer is negligible.

6. Base fluid and nanoparticles are in local thermal equilibrium.

The continuity and momentum Eqs. (9) and (10) are in the same form as the single-phase equations, but the energy Eq. (11) is modified with nanoparticle diffusion terms to reflect the Brownian and thermophoresis effects. These additional terms account for nanoparticle slip relative to the base fluid. Equation (12) is the volume fraction equation, which defines the advection portion and diffusion terms of the nanoparticle transport. Buongiorno also provides expressions for the diffusion constants of Brownian and thermophoresis effects as follows:

$$
\begin{aligned}
D_{B} & =\frac{k_{B} T}{3 \pi \mu d_{n p}}, \\
D_{T} & =\gamma \frac{\mu}{\rho_{f}} \phi .
\end{aligned}
$$

Here $k_{B}=1.38 \times 10^{-23} \mathrm{~m}^{2} \mathrm{~kg} \mathrm{~s}^{-2} \mathrm{~K}^{-1}$ is the Boltzmann constant, and $\gamma=0.26 \frac{k_{f}}{2 k_{f}+k_{n p}}$ is the proportionality factor. ${ }^{58}$ The effective viscosity and thermal conductivity have definitions similar to Eqs. (7) and (8). As suggested by Buongiorno, the eddy diffusivity of particles, $\epsilon_{P}$, is assumed to be of the same order as the eddy diffusivity of momentum. An assumption has been made that the nanoparticles are homogeneously entrained within the turbulent eddies of the continuous phase.

\section{Nanofluid properties}

The thermal conductivity of the nanofluid mixture can be determined using Maxwell's ${ }^{25}$ model, which was initially developed for macro-scale particle dispersion and later used in micro-scale particle dispersions. Under this approach, the effective thermal conductivity of a nanofluid is a function of conductivities of each constituent of the mixture and their 
corresponding volume fractions. In particular, the nanofluid thermal conduction is given by

$$
k_{n f}=\frac{k_{n p}+2 k_{f}-2 \phi\left(k_{f}-k_{n p}\right)}{k_{f}\left(k_{n p}+2 k_{f}+\phi\left(k_{f}-k_{n p}\right)\right)},
$$

where subscripts $(\cdot)_{f}$ and $(\cdot)_{n p}$ represent the relevant pure material values of the fluid and nanoparticles, respectively.

Einstein ${ }^{59}$ developed the first viscosity model to represent particle-fluid mixture viscosity for low particle concentrations. Later, Brinkman ${ }^{60}$ extended the validity of the model to $4 \%$ particle concentration and that model is now widely accepted as suitable for the effective viscosity of nanofluids,

$$
\mu_{n f}=\mu_{f} \frac{1}{(1-\phi)^{2.5}} .
$$

The other important quantities, such as $\rho_{n f},(\rho c)_{n f}$, and $\beta_{n f}$ can be calculated as follows:

$$
\begin{gathered}
\rho_{n f}=(1-\phi) \rho_{f}+\phi \rho_{n p}, \\
(\rho c)_{n f}=(1-\phi)(\rho c)_{f}+\phi(\rho c)_{n p}, \\
\beta_{n f}=(1-\phi) \beta_{f}+\phi \beta_{n p} .
\end{gathered}
$$

The pure thermo-physical properties of $\mathrm{Al}_{2} \mathrm{O}_{3}$ and water [the quantities denoted in subscripts of $\left.(\cdot)_{n p},(\cdot)_{f}\right]$, which are used to calculate nanofluid properties, are shown in Table I.

Throughout our study, we assume the same nanofluid correlations for both the $S_{P}$ and $T_{P}$ models.

\section{E. Boundary and operational conditions}

As with conventional fluid-flow simulations, the no-slip condition is assumed to apply at all wall boundaries. A zero condition is therefore imposed at the stator wall and, at the rotor wall, we have a tangential velocity set according to

$$
\boldsymbol{u}_{\text {rotor }}=\omega \boldsymbol{r}_{p} \times I
$$

Here $\boldsymbol{r}_{\boldsymbol{p}}$ is the position vector to a given point on the rotor surface, and $\boldsymbol{I}=(0,0,1)$ is the axial direction vector. The rotation rate $\omega$ is adjusted to achieve the required operational Reynolds number, $\operatorname{Re}_{\omega}=\omega r_{2}^{2} / v=1 \times 10^{5}$. The simulations are initialised with $T=300 \mathrm{~K}$ everywhere in the rotor-stator cavity domain, and the temperature boundary conditions are set to $T_{1}=300 \mathrm{~K}$ at all boundary surfaces except the stator, which is the only heat source of the cavity and has a constant surface temperature of $T_{2}=325 \mathrm{~K}$. This defines the volume fraction dependent thermal Rossby number of our simulations, $B=\beta_{n f}\left(T_{2}-T_{1}\right)$.

TABLE I. Thermo-physical properties of the constituent materials.

\begin{tabular}{lcc}
\hline \hline Property & $\mathrm{Al}_{2} \mathrm{O}_{3}$ & Water \\
\hline Density $(\rho)\left(\mathrm{kg} \mathrm{m}^{-3}\right)$ & 3970 & 996 \\
Viscosity $(\mu)\left(\mathrm{kg} \mathrm{m}^{-1} \mathrm{~s}^{-1}\right)$ & $\mathrm{N} / \mathrm{A}$ & $8.93 \times 10^{-7}$ \\
Heat capacity at constant pressure $\left(c_{p}\right)$ & 880 & 4177 \\
$\left(\mathrm{~J} \mathrm{~kg}^{-1} \mathrm{~K}^{-1}\right)$ & 42 & 0.61 \\
Thermal conductivity $\left(k_{T}\right)\left(\mathrm{W} \mathrm{m}^{-1} \mathrm{~K}^{-1}\right)$ & 30 & 0.385 \\
Diameter $\left(d_{p}\right)(\mathrm{nm})$ & $2.5 \times 10^{-5}$ & $2.1 \times 10^{-4}$ \\
Thermal expansion coefficient $(\beta)\left(\mathrm{K}^{-1}\right)$ & & \\
\hline \hline
\end{tabular}

In both the single-phase $\left(S_{P}\right)$ and the two-phase $\left(T_{P}\right)$ cases, nanoparticle volume fractions of $\phi=0,0.02,0.04,0.2$ are defined, and the effective nanofluid thermal conductivity, $k_{n f}$, and viscosity, $\mu_{n f}$, are calculated using the correlations given in Sec. II D. The notation $\phi=0.0$ refers to the situation of pure water. However, the formulation of the numerical scheme is such that $\phi=0.0$ may cause singularities and so $\phi=2 \times 10^{-5}$ is used to represent pure water. The recent investigations by Turkyilmazoglu ${ }^{43}$ and Mustafa et al. ${ }^{44}$ use the thermal conductivity and viscosity models at the relatively high volume fractions of $\phi=0.1$ and 0.2. Furthermore, the recent investigations of Bakar et al. ${ }^{61}$ and Avramenko et al. ${ }^{38}$ use the Buongiorno model to study the Brownian forces and thermophoresis effects of nanofluids with high volume fractions that are well beyond the prescribed limit of the thermophysical models. Following Turkyilmazoglu, ${ }^{43}$ we assume that the nanoparticle accumulations are significant when $\phi>0.2$ and that the thermal conductivity and viscosity models are valid up to $\phi=0.2$. Moreover, no numerical instabilities have been observed during the simulation, but the physical consequences will be discussed in Sec. III.

As there is no relative velocity between nanoparticles and fluid in the $S_{P}$ simulation, the initial values of the volume fraction do not change throughout the simulation time. However, in $T_{P}$ simulations, the volume fraction can change locally during the simulation, as governed by Eq. (12). This prompts the need for further boundary conditions at the walls (in addition to the initial conditions throughout the cavity) which can be constructed from the mass flux of nanoparticles. In particular, it assumed that the total mass flux $\boldsymbol{j}_{\boldsymbol{p}}$ due to the Brownian and thermophoresis forces is given by

$$
j_{p}=-\rho_{n p}\left[D_{B} \nabla \phi+D_{T} \frac{\nabla T}{T}\right],
$$

and, by imposing zero mass flux in the wall-normal directions, this becomes

$$
\left(\frac{\partial \phi}{\partial n}\right)_{\text {wall }}=-\frac{D_{T}}{D_{B} T}\left(\frac{\partial T}{\partial n}\right)_{\text {wall }},
$$

which is valid on the wall boundaries.

\section{RESULTS}

This section describes the momentum and heat transfer characteristics of rotor-stator cavities filled with $\mathrm{Al}_{2} \mathrm{O}_{3}$ nanofluids. We will use both the $S_{P}$ and $T_{P}$ transport models. First, velocity distributions obtained by both models for the $\phi=0.0$ case are validated against the previous results of Séverac et al. ${ }^{46}$ Secs. III A-III D will then describe the momentum and thermal characteristics of the $S_{P}$ and $T_{P}$ transport models for non-zero volume fractions, with the intention of understanding the dominant heat transfer modes of rotor-stator cavities filled with nanoparticles.

A sufficiently fine mesh is a crucial factor for a successful LES approach. As described in Eq. (3), the implicit filter length is proportional to the cube root of the local cell volumes, which means the smaller cell volumes can resolve the finer scales in the flow field. However, this approach is limited by the available computational resources for the simulation 
and maintaining the balance between the mesh resolution and computational resources are vital. We define a parameter, $Q=k / k_{\text {total }}$, to evaluate the suitability of our current mesh configuration. Here, $k_{\text {total }}=k+k_{s g s}$ is the total turbulent kinetic energy, and $k=0.5\left(\overline{u_{r}^{\prime} u_{r}^{\prime}}+\overline{u_{\theta}^{\prime} u_{\theta}^{\prime}}+\overline{u_{z}^{\prime} u_{z}^{\prime}}\right)$ and $k_{s g s}=1 / 2 \operatorname{tr}\left(\tau_{s g s}\right)$ are the resolved and sub-grid scale components of the turbulent kinetic energy. Figures 2(a)-2(d) present top and side views of the $Q$ contours for the $S_{P}$ and the $T_{P}$ simulations at the nanoparticle volume fraction of $\phi=0.0$. These contours suggest that in most of the regions, $Q$ values are close to the unity, but the area near the stator and rotor hub shows high values of $k_{\text {sgs }}$. Figure 2(e) shows the percentage of cells versus $Q$ values and this histogram was constructed by considering the entire simulation domain of the rotor-stator cavity. This result suggests that the $80 \%$ of the cells in our simulation domain are $Q>0.975$.

For the purposes of presentation, the radial and axial distances are non-dimensionalised as $r^{*}=\frac{\left(r-r_{1}\right)}{\left(r_{2}-r_{1}\right)}$ and $z^{*}=\frac{z}{h}$, respectively. The mean resolved radial and tangential velocity components are non-dimensionalised as $U_{r}^{*}, U_{\theta}^{*}=\frac{U_{r}}{r \omega}, \frac{U_{\theta}}{r \omega}$, and the radial and tangential turbulence intensity components are normalised using $\sqrt{R_{\theta \theta}}=\frac{\sqrt{\overline{u_{\theta}^{\prime} u_{\theta}^{\prime}}}}{(r \omega)}$ and $\sqrt{R_{r r}}=\frac{\sqrt{u_{r}^{\prime} u_{r}^{\prime}}}{(r \omega)}$. All the velocity contours are usually normalised by the $U_{\theta, \max }=r_{2} \omega$. The full axial extent of the cavity is then given by the range $z^{*}=[0,1]$ with the lower and upper bounds representing the rotor and stator surfaces, respectively. All axial plots are extracted at the mid-radial position of the cavity, $r^{*}=0.5$, where the effects of the finite cavity is minimal. All mean velocity components and turbulence intensity profiles are averaged over a sufficient time interval after the simulations reach a statistically steady state. Furthermore, both mean and instantaneous quantities are averaged over the azimuthal direction to obtain smoother distribution profiles.

\section{A. Validations of $S_{P}$ and $T_{P}$ nanofluid transport models}

Figure 3 shows the mean radial and tangential velocity profiles at $r^{*}=0.5$ for $\phi=2 \times 10^{-5}$. Both the $S_{P}$ and $T_{P}$ models are seen to lead to similar radial and tangential velocity profiles. This is of no surprise because the $T_{P}$ model effectively limits to the $S_{P}$ model as $\phi \rightarrow 0$. The entrainment coefficient,

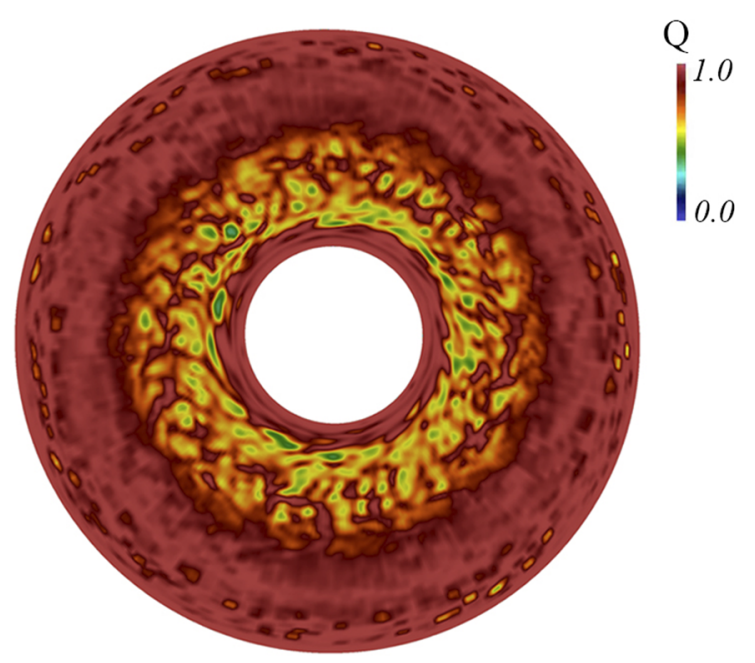

(a)

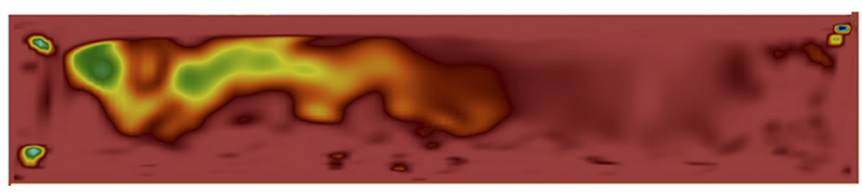

(c)

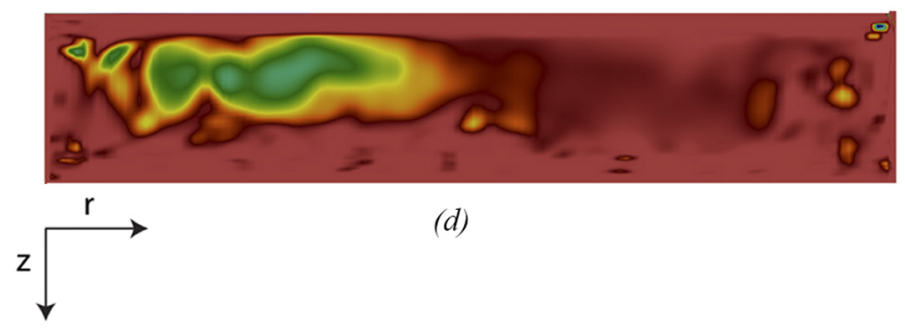

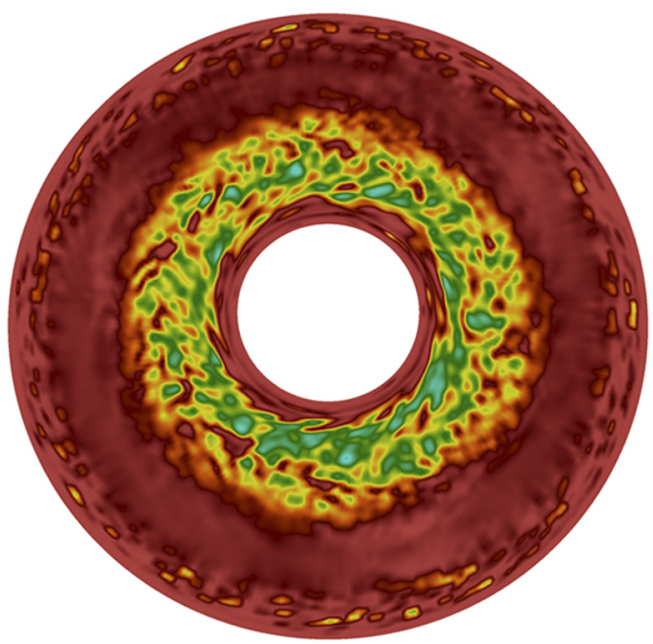

(b)

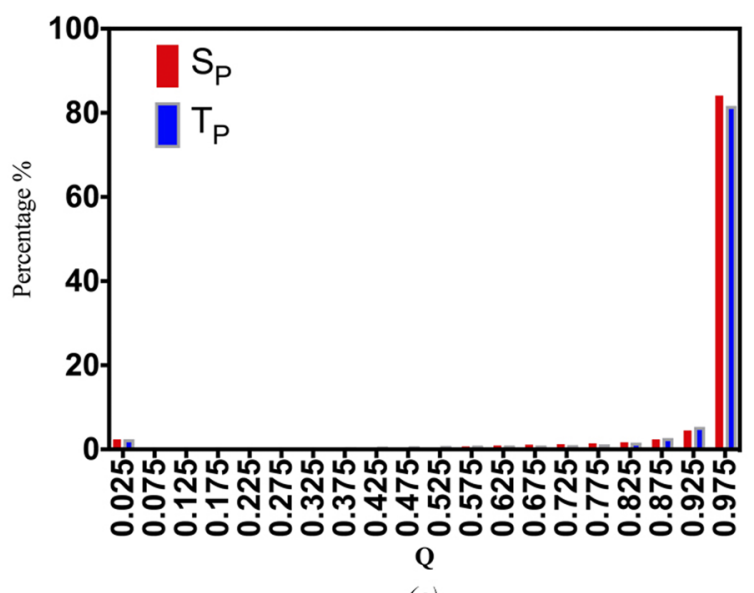

(e)

FIG. 2. The contours of $Q$ and the histogram of the rotor stator cavity at $\operatorname{Re}_{\omega}=1 \times 10^{5}$ for $\phi=0.0$. (a) top view of the contours at the mid-section of the cavity for $S_{P}$ model, (b) top view of the contours at the mid-section of the cavity for $T_{P}$ model, (c) side view of the contours at the $r-z$ plane of the cavity for $S_{P}$ model, (d) side view of the contours at the $r-z$ plane of the cavity for $T_{P}$ model, and (e) $Q$ vs percentage of cells. 


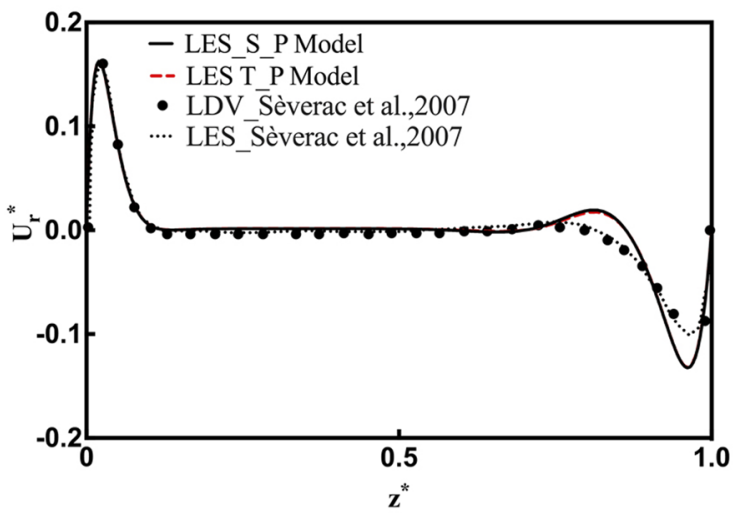

(a)

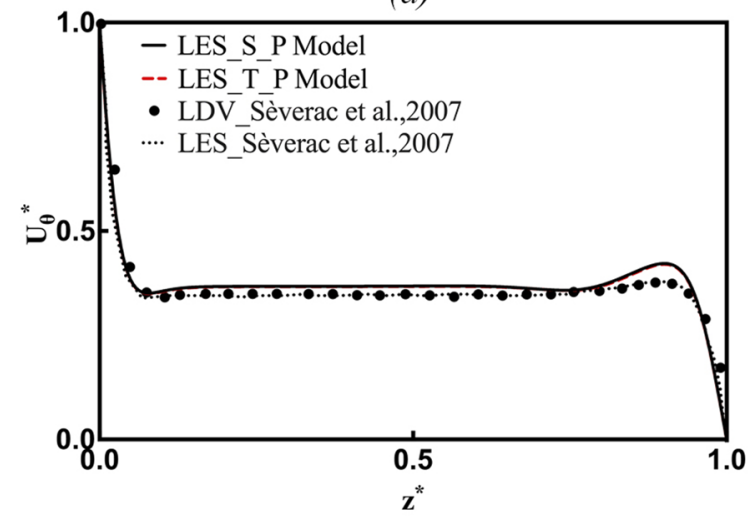

(b)

FIG. 3. The axial distributions of mean velocity profiles for pure water, $\phi=0.0, r^{*}=0.50, R e_{\omega}=1 \times 10^{5}$, (a) radial velocity, (b) tangential velocity.

$K=\frac{U_{\theta, c o r e}}{U_{\theta, \text { disk }}}$ indicates the strength of rotation in the core region of the cavity, and both models lead to $K \approx 0.36$. Under similar conditions, Séverac et al. ${ }^{46}$ obtained $K \approx 0.35$ using both numerical and experimental approaches.

Figure 4 gives the radial and tangential turbulence intensity profiles arising from each simulation model. Both models are seen to under-predict the turbulence intensity profiles as compared to the previous experimental and LES results of Séverac et al., but near the stator boundary layer, the tangential turbulence intensity profile over-predicts when it is compared to the experimental tangential turbulence intensity profile of Séverac et al. The locations of peaks of the turbulence intensity profiles show agreement with previous experimental and LES results. Moreover, we note that the $S_{P}$ and $T_{P}$ simulations show very similar turbulence intensity profiles across the cavity, demonstrating consistency between the two models.

\section{B. Single-phase simulations of rotor-stator nanofluids}

Here we consider the momentum and heat transfer characteristics of rotor-stator cavities filled with nanoparticles at volume fractions $\phi=0.0,0.02,0.04$, and 0.2 . This section exclusively uses the $S_{P}$ model and, in line with Sec. III A, all the velocity and thermal distributions are obtained at $r^{*}=0.5$.

Figures 5(a)-5(d) show the mean radial velocity profiles within the rotor and stator boundary layers. At lower volume fractions, no significant changes can be observed in the
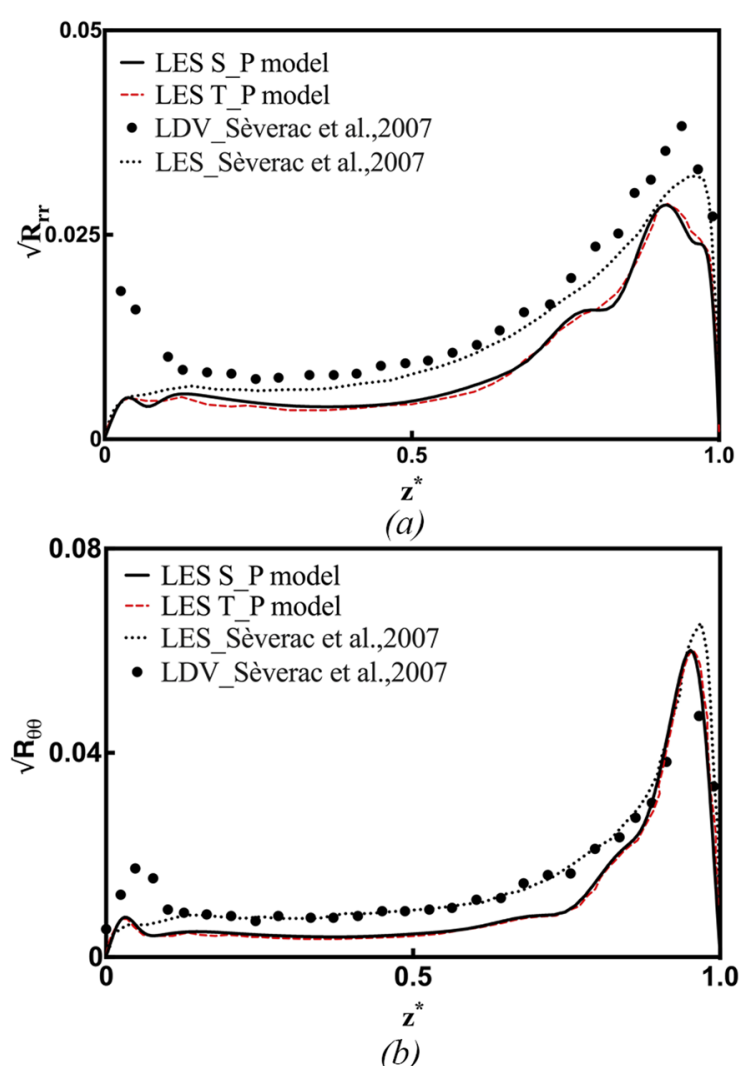

FIG. 4. The axial distributions of turbulence intensity profiles for pure water, $\phi=0, r^{*}=0.50, \operatorname{Re}_{\omega}=1 \times 10^{5}$, (a) radial turbulence intensity, (b) tangential turbulence intensity.

boundary layers, but higher volume fractions are seen to increase the radial fluid velocity. A similar trend is observed in the tangential velocity profiles of the rotor boundary layer in Fig. 5(c). However, in Fig. 5(d), the stator tangential velocity component is seen to reduce with increased volume fractions.

The axial velocity profiles for rotor and stator boundary layers, shown in Fig. 6, demonstrate greater axial entrainment into both boundaries as the concentration of the nanoparticle increases. Such behavior can be explained as a result of the continuity of the flow field. That is, the higher rate of fluid displacement in the radial and tangential directions demands a higher rate of fluid entrained from the axial direction of the cavity to replace it. The trend is less pronounced in the stator boundary later, but Fig. 6(b) shows a markedly increased velocity at $\phi=0.2$. Intuitively, a higher flow into the rotor boundary should result from the outflow of the stator boundary. However, mass transfer processes in rotor-stator flows are often complicated by the presence of the rotor hub and outer walls along which bulk mass transfer between the rotor and stator can occur. The previous theoretical investigation of Turkyilmazoglu ${ }^{43}$ explained the behavior of the velocity components near a single rotating disk which confirms our observations of the mean velocity components for $\mathrm{Al}_{2} \mathrm{O}_{3}$ nanofluids. Regardless of the volume fractions, the effects of nanoparticles on the mean radial and tangential distributions are highly localised to the near-wall regions and the velocity distributions eventually collapse onto each other at distances away from the wall. This results in similar behavior in the core region of the cavity for all $\phi$. 

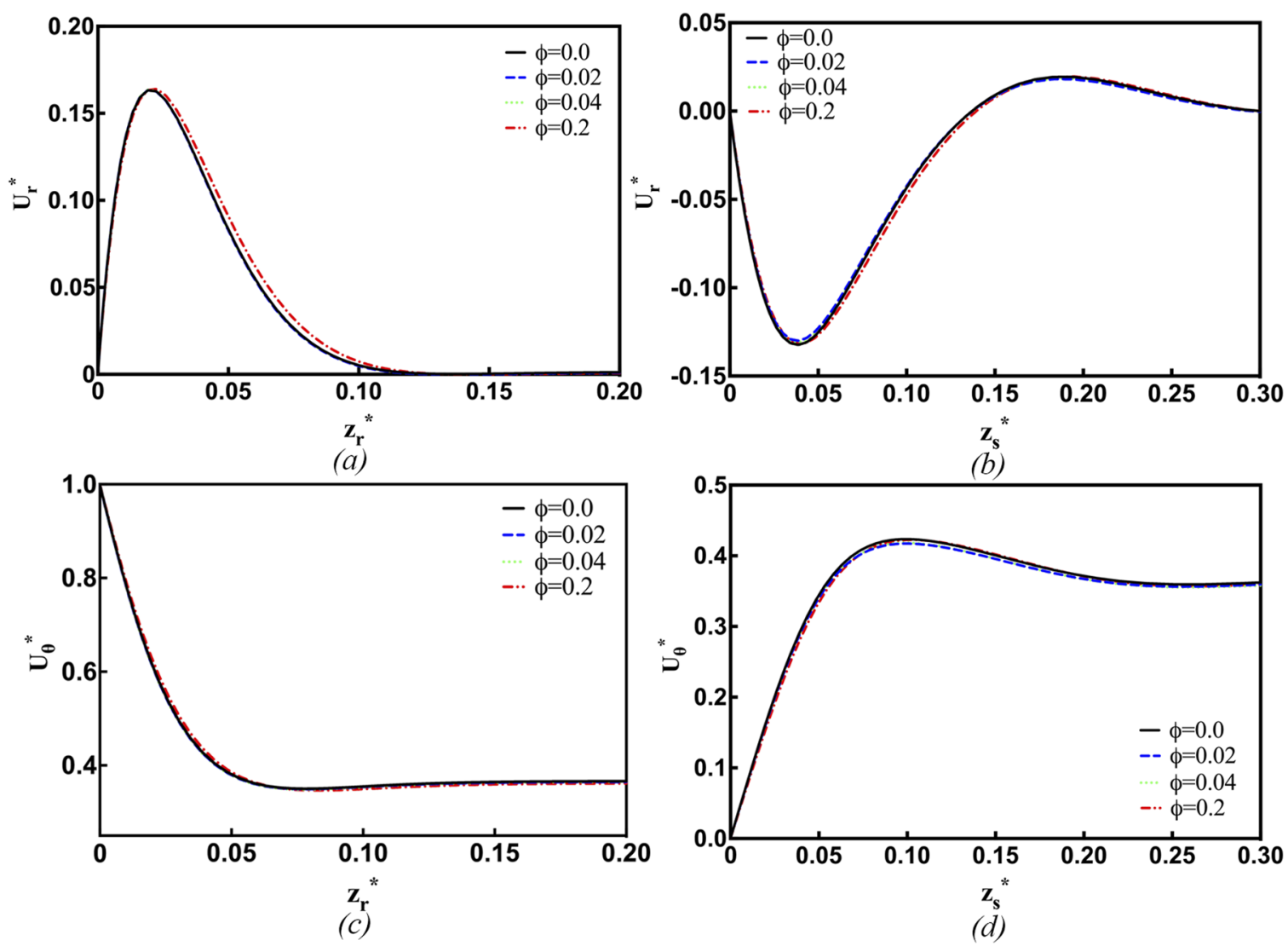

FIG. 5. A comparison of mean radial and tangential velocity profiles at $r^{*}=0.50$ for different nanoparticle volume fractions for the $S_{P}$ model, $R e_{\omega}=1 \times 10^{5}$, (a) radial velocity profiles of the rotor boundary layer, (b) radial velocity profiles of the stator boundary layer, (c) tangential velocity profiles of the rotor boundary layer, and (d) tangential velocity profiles of the stator boundary layer.
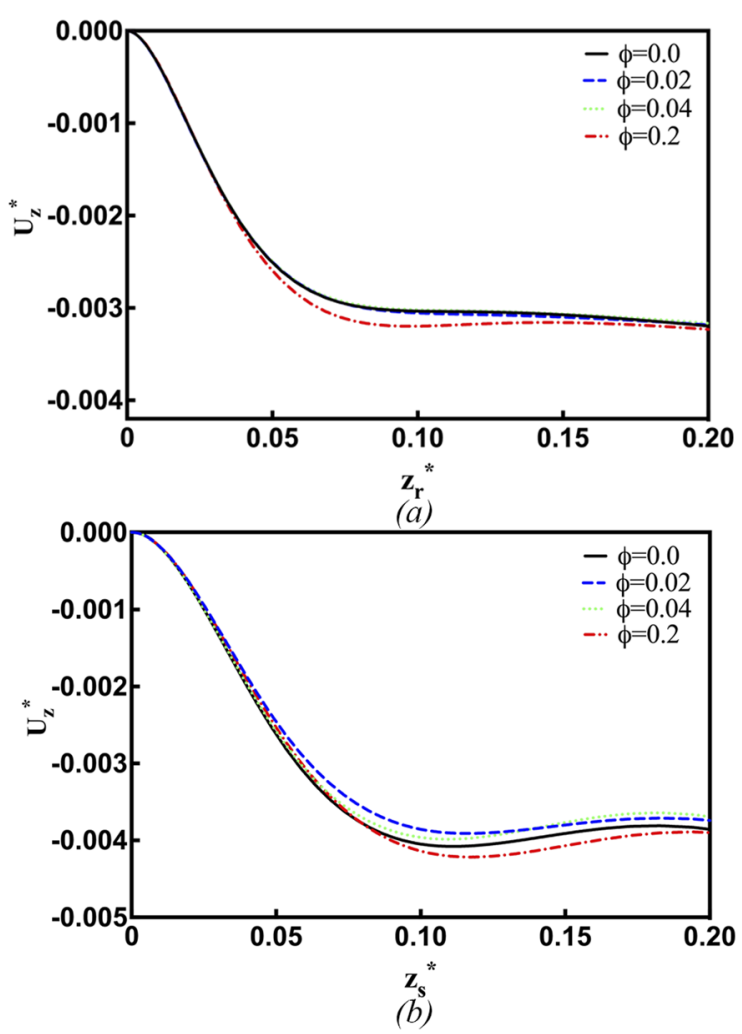

FIG. 6. A comparison of mean axial velocity profiles at $r^{*}=0.50$ for the $S_{P}$ model, $R e_{\omega}=1 \times 10^{5}$, (a) rotor boundary layer, (b) stator boundary layer.
Figure 7 describes the normalised axial velocity distributions in the $r-z$ plane, and it shows a train of vortex structures initiated in the stator boundary. In rotor-stator cavities, fluid transfer between the rotor and stator boundary layers occur over the outer walls and the inner rotor hub of the cavity. The colder fluid flow in the rotor rises to higher $z$ values near the outer wall, and hotter fluid near the stator is transported pair the inner hub. This flow circulation process helps to accelerate the heat transfer between the two boundary layers of the cavity. The mean axial velocity profiles (not shown here) evidence that the velocity of the bulk fluid flows near the rotor hub and outer wall increases as the nanoparticle volume fractions increases.

For the sake of completeness, instantaneous normalised tangential velocity components at the rotor $\left(z^{*}=0.025\right)$ and stator $\left(z^{*}=0.975\right)$ boundaries for different volume fractions are shown in Fig. 8. As described in Fig. 5, higher volume fractions are predicted to produce a marginal increase in the velocity components in the rotor boundary layer and a decrease in velocity in the stator boundary layer. Apart for nanofluid related effects, these boundary layers comply with the typical behavior of rotor-stator boundary layers at $R e_{\omega}=1 \times 10^{5}$, in which rotor boundary layers are mostly laminar, while stator boundary layers show transient turbulent structures. Previous investigations by Séverac et al. ${ }^{46}$ and Makino et al. ${ }^{47}$ describe the isothermal boundary-layer behavior of rotor-stator cavities. Bridel-Bertomeu ${ }^{62}$ investigated cylindrical and annular 


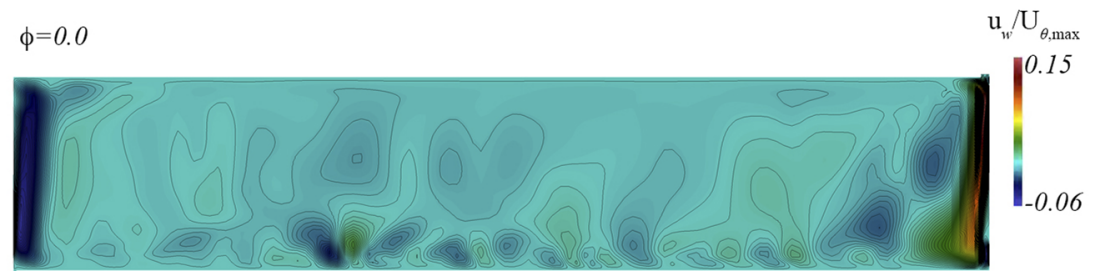

(a)

$\phi=0.04$

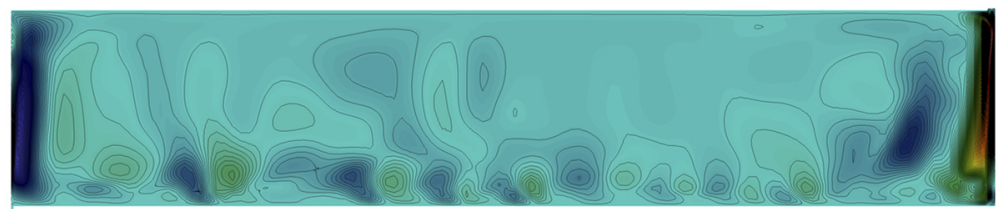

(b)

$\phi=0.2$

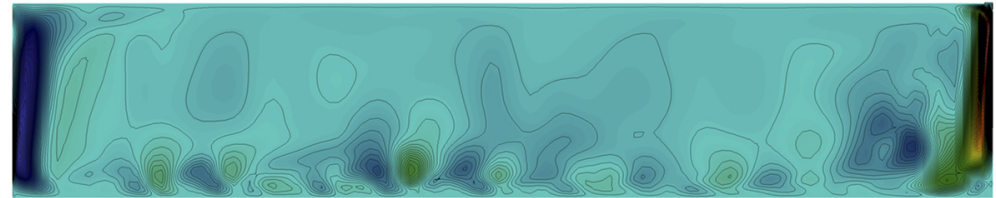

$z \stackrel{r}{\longrightarrow}$

(c)
FIG. 7. Instantaneous normalised axial velocity contours in the $r-z$ plane, (a) $\phi=0.0$, (b) $\phi=0.04$, and (c) $\phi=0.2$. rotor-stator cavities with curvature radii $R_{m}=1.0$ and 1.8 and aspect ratios $G=0.2$ and 1.18 at the similar operational Reynolds number of $R e_{\omega}=1 \times 10^{5}$. There a wall-resolved
LES method was used to study the instability patterns in the boundary layers. The study did an excellent job of visualising the transient instability patterns using fluctuation velocity

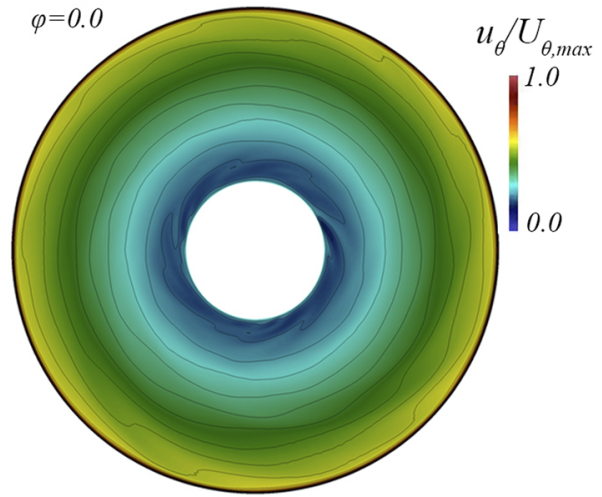

(a)

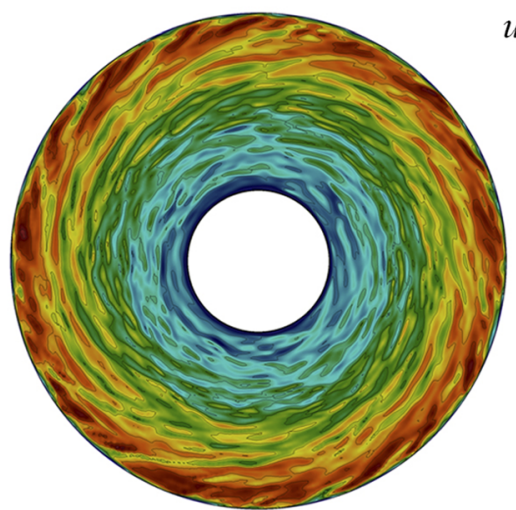

(d)

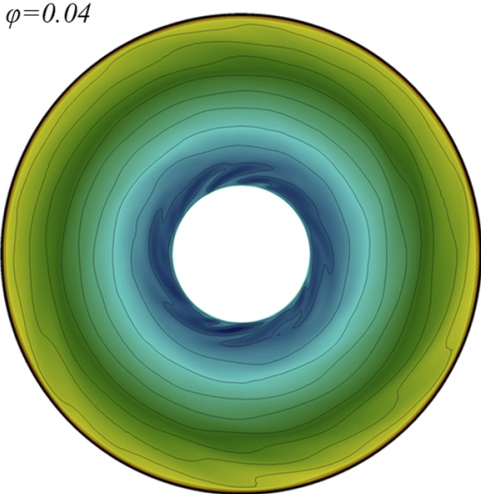

(b)
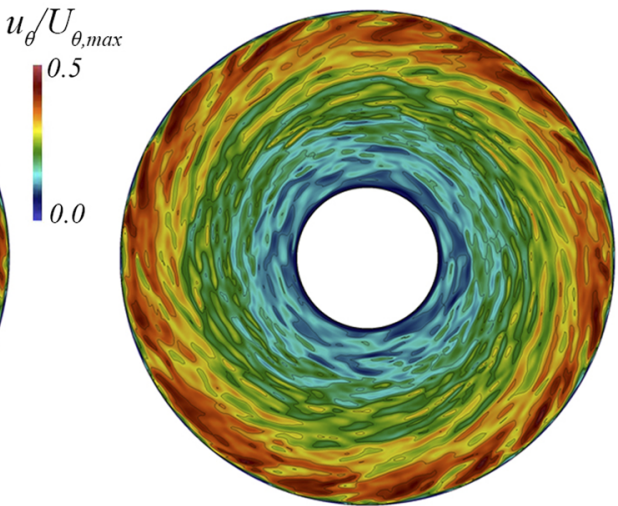

(e)

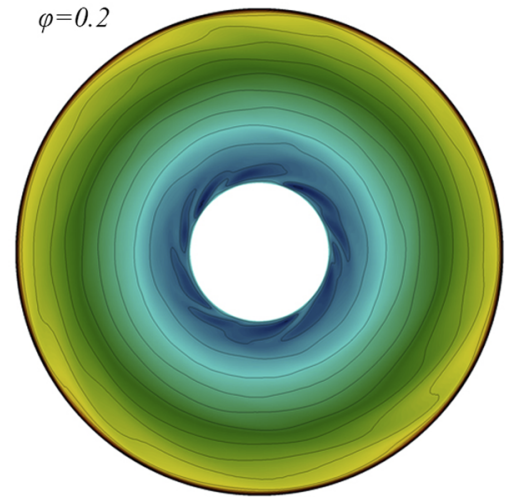

(c)

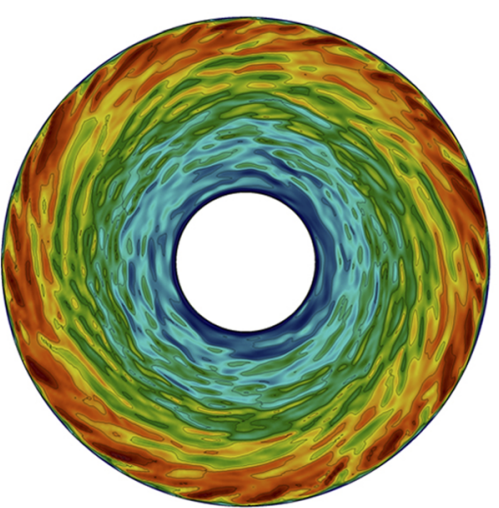

(f)

FIG. 8. Instantaneous normalised tangential velocity contours, at rotor boundary, $z^{*}=0.025$, (a) $\phi=0.0$, (b) $\phi=0.04$, and (c) $\phi=0.2$; at stator boundary, $z^{*}=0.975$, (d) $\phi=0.0$, (e) $\phi=0.04$, and (f) $\phi=0.2$. 
components. Moreover, our recent publication ${ }^{63}$ visualised the development of instability patterns on the rotor boundary layer of an identical rotor-stator cavity using a vortex identification method, ${ }^{64}$ and the outcomes at $R e_{\omega}=1 \times 10^{5}$ have been compared with a higher Reynolds number case of $R e_{\omega}=4 \times 10^{5}$.

Figure 9 shows radial and tangential turbulence intensity profiles at $r^{*}=0.50$ for different volume fractions. It can be seen that the radial turbulence intensity profiles are more sensitive to changes in volume fractions. The tangential turbulence intensity profiles show some effects but in a less pronounced manner. At the highest volume fraction, $\phi=0.2$, there is a visible reduction in turbulence intensity profiles adjacent to the wall and in the core region of the cavity in both the radial and tangential distributions. By contrast, at $\phi=0.02$ and 0.04 we find subtle increases as compared to pure water at these same axial locations. While this change in behavior could be physical, it could also be a consequence of either the incompatibility of the viscosity model or nanoparticle agglomeration at higher volume fractions. A further experimental investigation could confirm the exact cause of the reduction of turbulence intensity profiles for $\phi=0.2$. These detailed questions aside, an increase in the nanoparticle volume fraction is generally seen to result in higher turbulence intensity profiles across all axial locations within the cavities.

Previously, Hu et al. ${ }^{65}$ conducted an LES-Lagrangian based numerical investigation to study the transport properties of a nanofluid based on $\mathrm{Cu}$ and $\mathrm{SiO}_{2}$ nanoparticles, and observed that nanofluids produce higher turbulence intensity profiles at $\phi=0.01$ compared to pure water simulations.
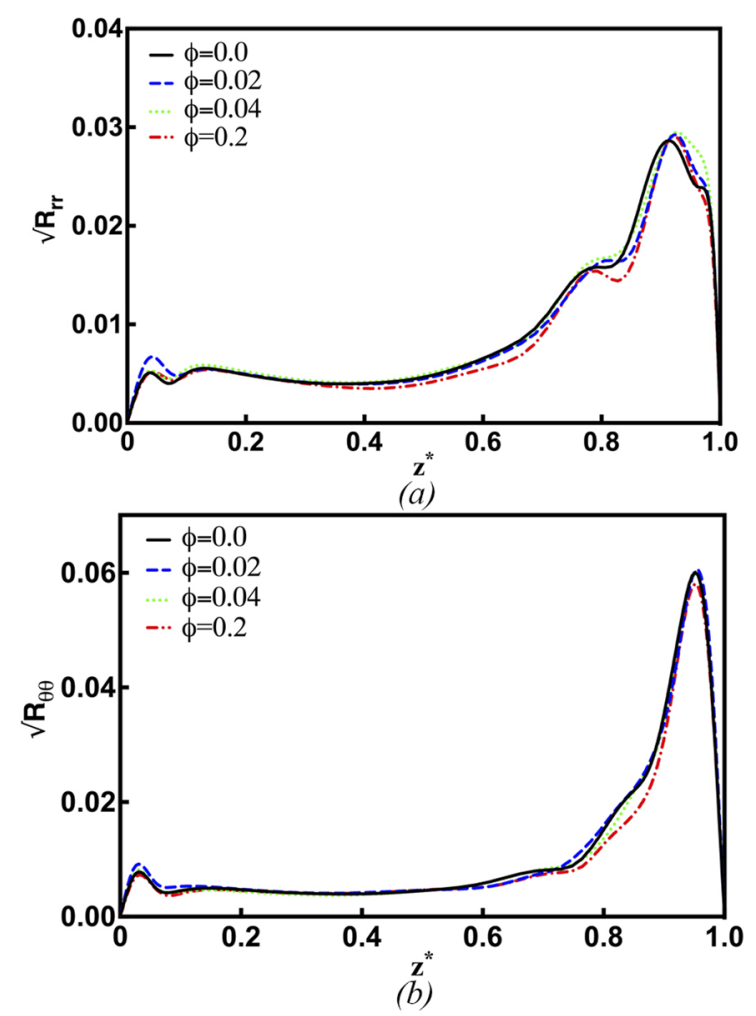

FIG. 9. A comparison of the turbulence intensity profiles for different nanoparticle volume fractions, $r^{*}=0.50$ for the $S_{P}$ model, $R e_{\omega}=1 \times 10^{5}$, (a) radial turbulence intensity profiles, (b) tangential turbulence intensity profile.
Whereas, Ghaffari et al. ${ }^{66}$ reported a reduction in turbulence intensity profiles with increased the Grashof number $(\mathrm{Gr})$ in their simulations of horizontal curved pipes based on a two-phase modeling approach with slip velocities. These simulations highlight the uncertain nature of the true effects of increased volume fraction in the absence of experimental data. However, it is important to note that both examples have bulk fluid flow in a streamline direction, whereas rotor-stator flows have a fluid motion induced by rotation of the rotor. This difference may or may not be important.

The mean velocity and turbulence intensity profiles of the rotor-stator cavities have given a visible response to the changes in nanoparticle volume fractions, but, in both cases, these changes are subtle [with the exception of Fig. 9(a)]. Hence they may not give significant contributions to the convective heat transfer process. These results suggest that the addition of nanoparticles to a base fluid has a less significant effect on momentum transport for rotor-stator applications, which is a primary motivation of using nanofluids in heat transfer applications instead of mixtures based on micro-sized particles. However, the current investigation only concerns $\mathrm{Al}_{2} \mathrm{O}_{3}$ nanoparticles, and the investigation of Turkyilmazoglu ${ }^{43}$ concerning the rotating disk suggests that nanofluids based on $\mathrm{Cu}$, $\mathrm{CuO}$, or $\mathrm{Ag}$ can have more pronounced effects on momentum transport. Moreover, we suspect that many of the characteristics of the mean velocity and turbulence intensity distributions are affected by the finite nature of the cavity, and selecting the distributions at the mid-section of the cavity has only discounted the issue to a certain extent.

Despite the subtle effects of nanoparticle volume fractions on momentum transport, Fig. 10 shows that test cases with higher volume fractions occupy elevated temperature distributions, especially in the core region of the cavity. This initial result suggests that rotor-stator cavities filled with nanofluids may have superior heat transfer capabilities compared to conventional cavities filled with pure water. Figure 11 shows instantaneous temperature contours in the $r-z$ plane for different volume fractions. The central core region of the cavity has higher temperature values than the outer core region, where the temperature distributes more quickly due to the higher rotation rates and entrainment coefficients. The hot fluid stream on the rotor hub is the main passage of the heat transfer from the stator to the rotor, and the rotating motion of the hub spreads the

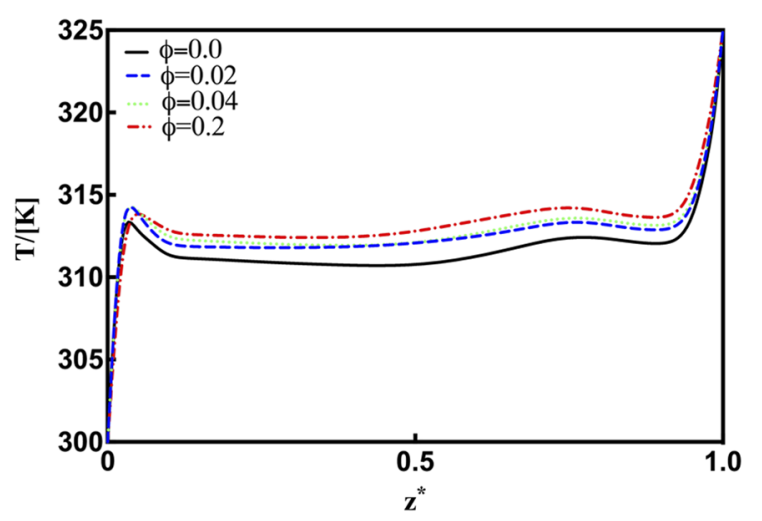

FIG. 10. A comparison of mean temperature distributions for different nanoparticle volume fractions, $r^{*}=0.50$ for the $S_{P}$ model, $R e_{\omega}=1 \times 10^{5}$. 


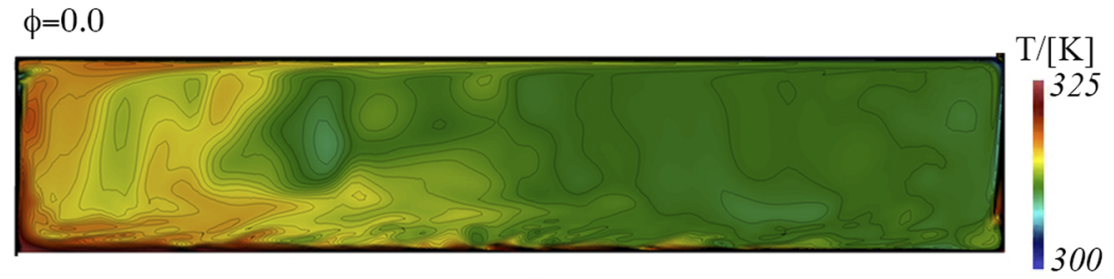

(a)

$\phi=0.04$

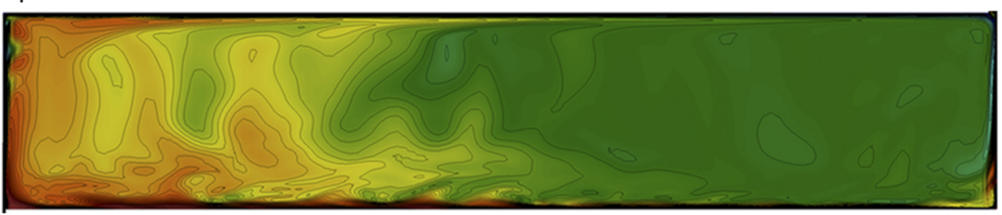

(b)

$\phi=0.2$
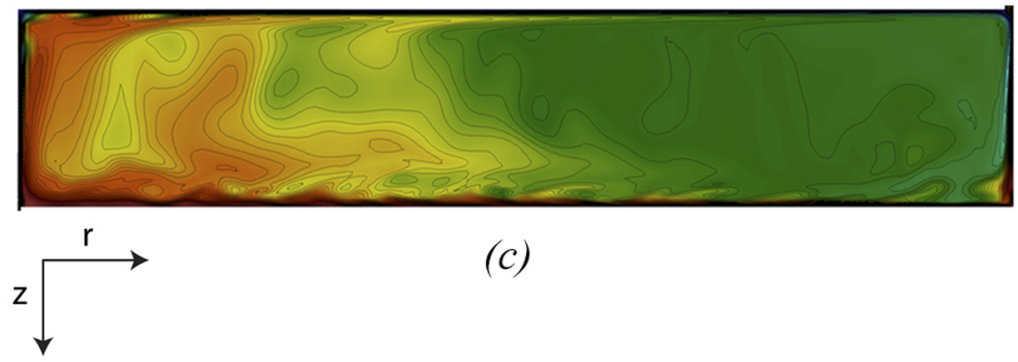

(c)
FIG. 11. A comparison of instantaneous temperature contours of $S_{P}$ simulations at the $r-z$ plane, $R e_{\omega}=1 \times 10^{5}$, (a) $\phi=0.0$, (b) $\phi=0.04$, and (c) $\phi=0.2$. heat to the central core region of the cavity. From the temperature contours, it is clear that cavities with higher nanoparticle volume fractions show a greater spread in temperature distributions and lead to relatively large-scale temperature intrusions near the rotor hub and the stator.

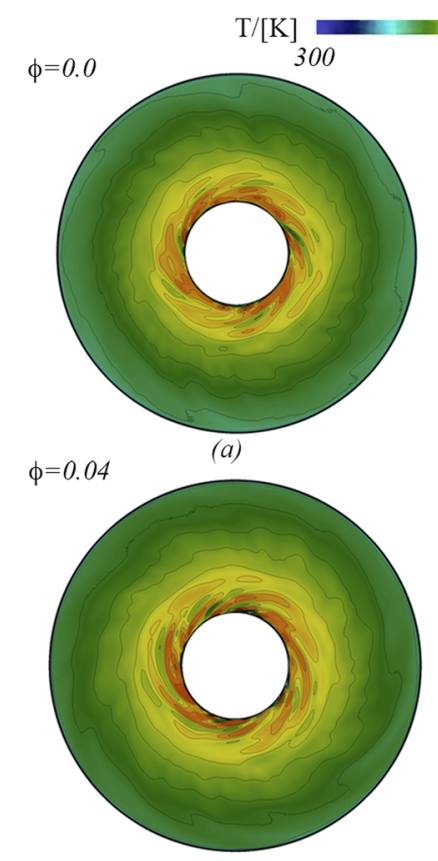

(c)

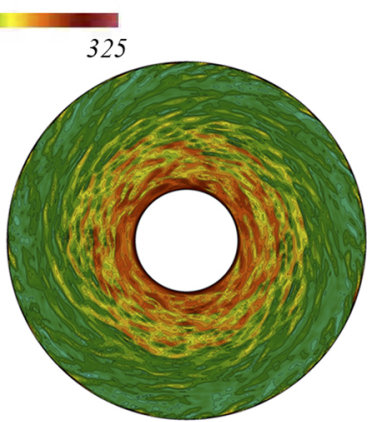

(b)

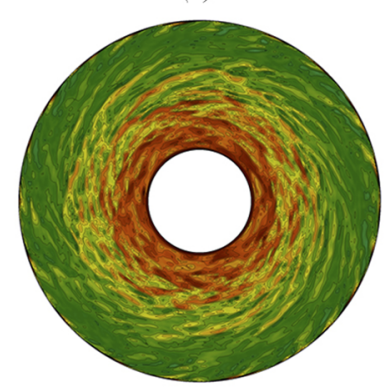

(d)
FIG. 12. A comparison of instantaneous temperature contours at the two boundary layers (a) rotor boundary, $\phi=0.0$, (b) stator boundary, $\phi=0.0$, (c) rotor boundary, $\phi=0.04$, and (d) stator boundary, $\phi=0.04$ for different nanoparticle volume fractions for the $S_{P}$ model, $R e_{\omega}=1 \times 10^{5}$.
The top view of the temperature contours of the rotor (left column) and stator (right column) are shown in Fig. 12. These further confirm the role of the rotor hub in spreading the heat toward the core region of the cavity. At the rotor boundary, there are large scale heat structures at the rotor hub, and, at the stator boundary, the heat structures become even smaller in size. Even though these structures can be described as "heat structures" they are disturbance structures, which are present over all typical turbulent stators (see Fig. 8). We see that such disturbances play an essential role in rotor-stator heat transfer by spreading the hot fluid streams throughout the cavity.

\section{Two-phase simulations of rotor-stator nanofluids}

This section describes the momentum and heat transfer characteristics for our two-phase, $T_{P}$, model. As already discussed, the main difference between $S_{P}$ and $T_{P}$ models are that the latter allows for a relative velocity between the nanoparticles and the base fluid. This results in a volume fraction distribution inside the cavity, instead of a time-independent homogeneous volume fraction.

A set of simulations with initial volume fractions identical to those considered in the $S_{P}$ simulations have been conducted for the $T_{P}$ model, and those simulations are identified by their initial volume fraction amounts (hereafter, we denote this as the inceptive volume fraction $\phi_{t_{0}}$ ). Interestingly, Fig. 13 shows that the momentum-transport behavior found for the $T_{P}$ model is almost identical to that found using the $S_{P}$ model. We do not, therefore, discuss these results any further and instead the reader is referred to the discussions of mean velocity and turbulence intensity transport made in Sec. III B, particularly Figs. 5 and 9. 

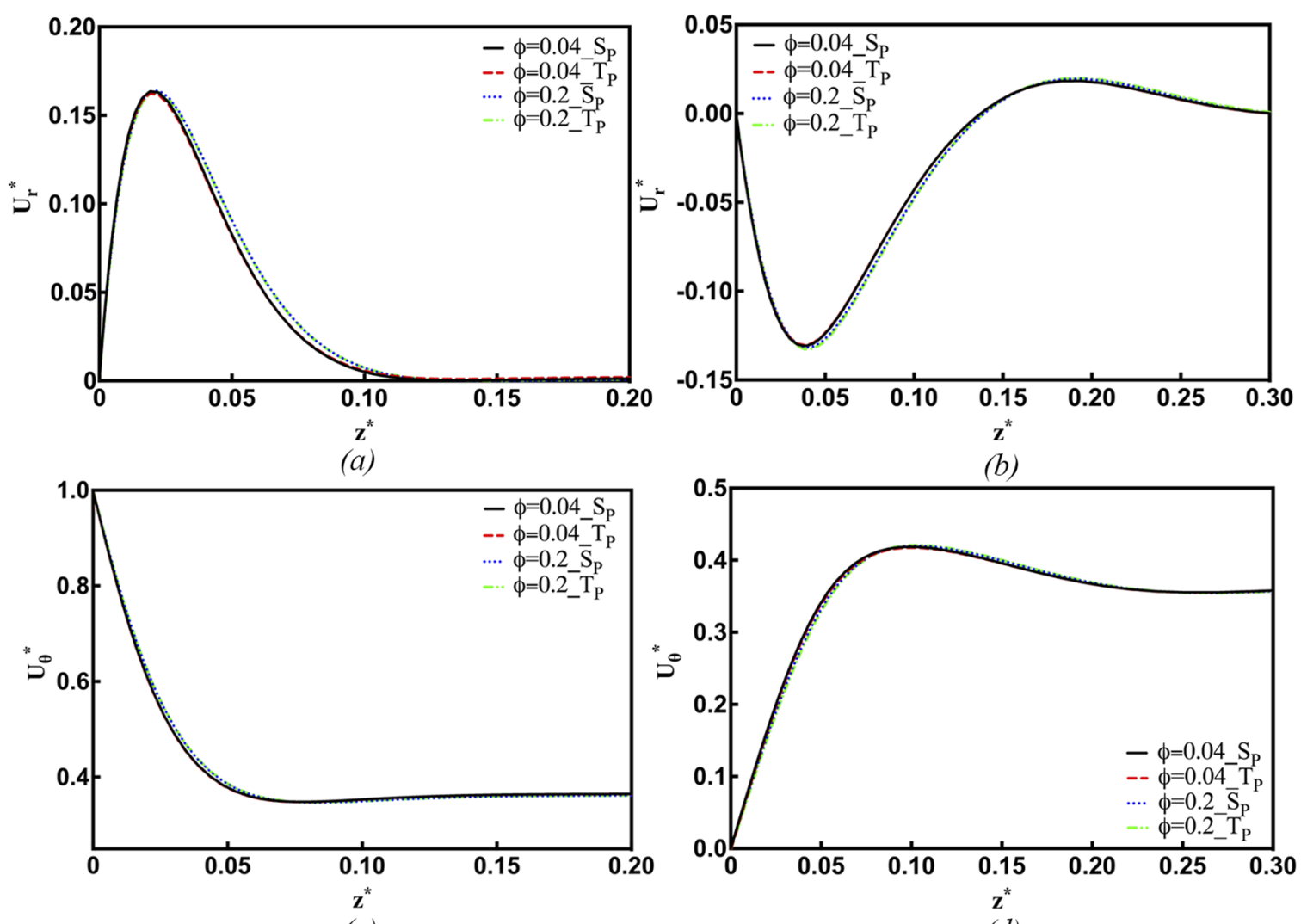

FIG. 13. A comparison of mean radial and tangential velocity profiles for the $S_{P}$ and the $T_{P}$ models at different nanoparticle volume fractions, $r^{*}=0.50$, $\operatorname{Re}_{\omega}=1 \times 10^{5}$.

In contrast to the momentum-transport behavior, significant differences in the heat transfer predictions are found between the two models. The mean temperature distributions across the axial domain arising from both models are shown in Fig. 14. We note differences in the thermal distributions across the cavity, which signifies that the $T_{P}$ modeling approach has altered the underlying thermal transport mechanism of the simulated rotor-stator cavity. The temperature distributions suggest that the $T_{P}$ model reduces the efficiency of the heat dispersion when compared to the $S_{P}$ modeling approach. At first glance, this suggests that the relative straights of the thermophoresis forces dominate the Brownian effects in the heat

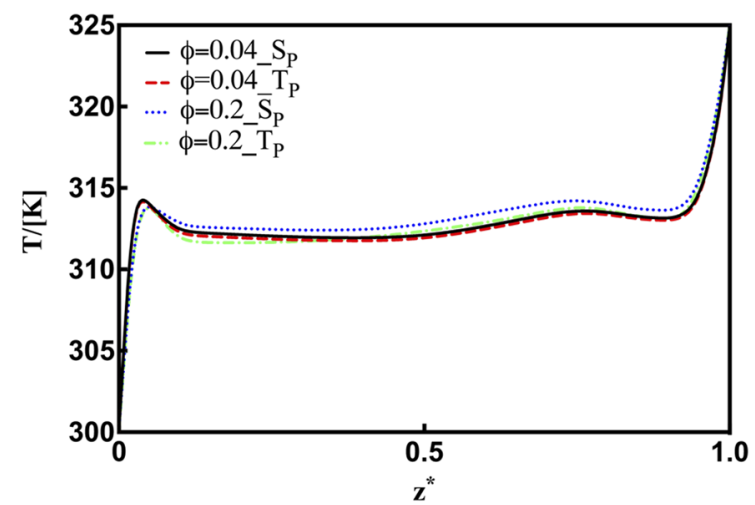

FIG. 14. A comparison of mean temperature distributions for the $S_{P}$ and the $T_{P}$ models at different nanoparticle volume fractions, $r^{*}=0.50$, $R e_{\omega}=1 \times 10^{5}$. transfer mechanisms of rotor-stator cavities filled with nanofluids. This motivates further investigation but is beyond the scope of this paper.

Figure 15 shows the instantaneous distribution of the normalised local volume fraction, $\alpha=\phi / \phi_{t_{0}}$, in the $r-z$ plane arising from the $T_{P}$ model for $\phi_{t_{0}}=0.2$. Note that the value is scaled against the inceptive volume fraction $\phi_{t_{0}}$. The figure shows that nanoparticles tend to aggregate just above the stator boundary layer and beside the rotor hub, where most of the hot fluid transport occurs. The core region of the cavity is relatively unaffected, but some areas, which occupy hot fluid, have lower concentrations of nanoparticles. These observations again suggest that thermophoresis effects play a significant role in the heat transfer of rotor-stator cavities.

Figure 16 shows normalised volume fraction contours extracted near the laminar sublayer of each boundary layer. These contours evidence that there is a layer of nanoparticles present over the rotor wall (except for a very small area near the rotor boundary hub), but, at the same time, nanoparticles are displaced away from the stator boundary layer. The displacement of the nanoparticles in the stator layer is significant at higher radial positions, and these displaced volume fractions could result in the thick layer of nanoparticles in the outer region of Fig. 15.

Figure 17 shows the spatial variations of normalised volume fraction, produced for the case of $\phi_{t_{0}}=0.2$. Figure 17(a) represents the variation of normalised volume fractions in the wall normal direction at the different radial positions. It suggests higher nanoparticle concentrations at the inner regions 


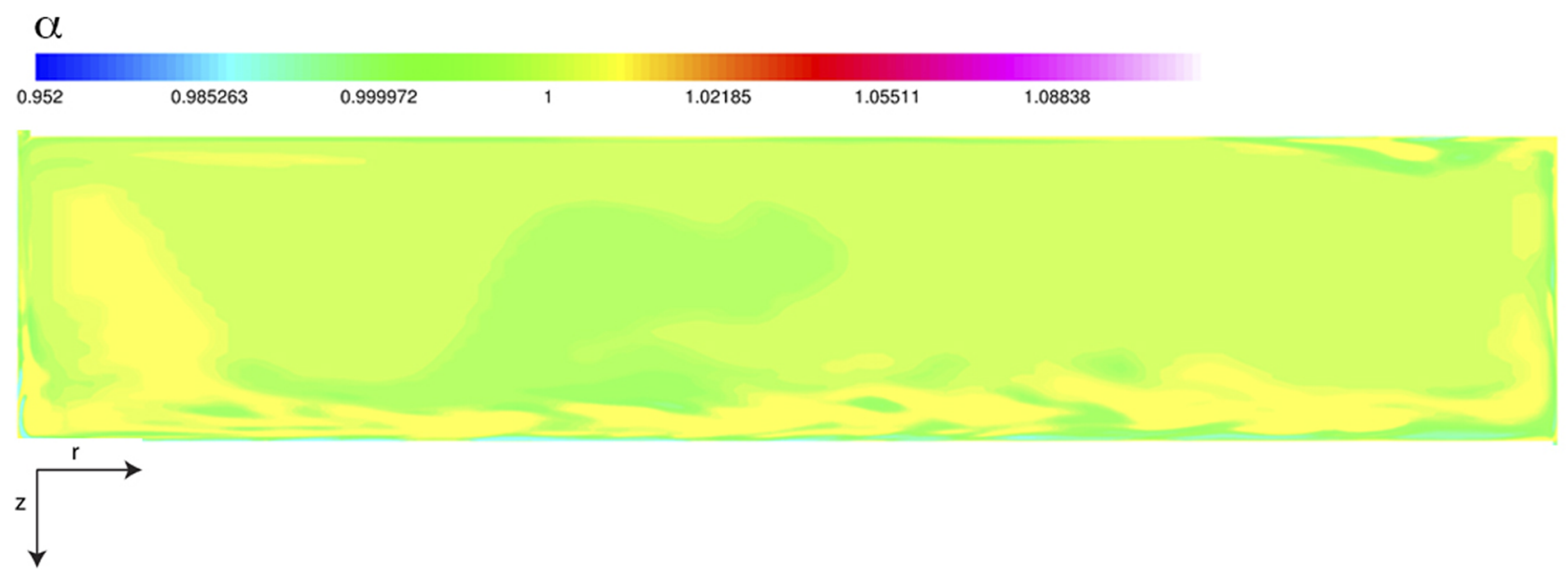

FIG. 15. An instantaneous distribution of the normalised local volume fraction contour $\alpha$ for a $T_{P}$ simulation, $\phi=0.2, R e_{\omega}=1 \times 10^{5}$ at the $r-z$ plane.

of the rotor boundary layer, and relatively low particle concentrations (but still higher than the $\phi_{t_{0}}$ ) at the mid and outer regions of the rotor boundary layer. The normalised volume fractions in the core region of the cavity are nearly constant with $\alpha \approx 1$. Furthermore, the stator boundary layer has lower nanoparticle concentrations with $\alpha<1$, particularly at larger radial positions. Figure 17(b) further confirms this variation and shows that high nanoparticle concentrations occur in both the rotor and the stator boundary layers at all radial locations where $r^{*}<0.47$.

The above description suggests a plausible reason for the lower mean temperature distributions arising from $T_{P}$ models. That is, while the nanoparticles have a beneficial effect on heat transfer, they do not in practice remain evenly distributed throughout the cavity as the $S_{P}$ model assumes. The $S_{P}$ model oversimplifies the true behavior of the nanoparticles
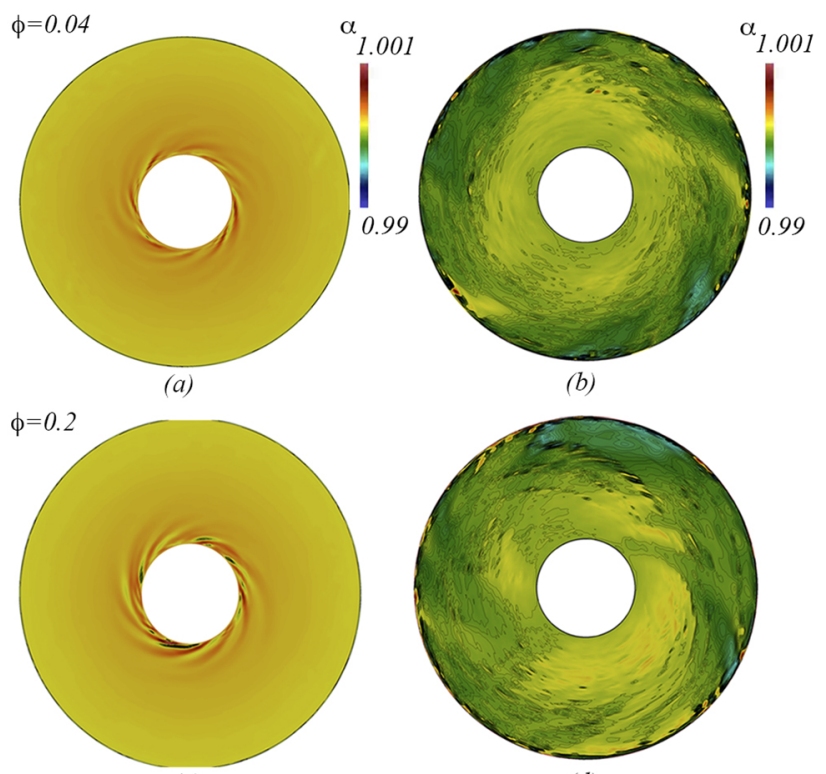

(c)

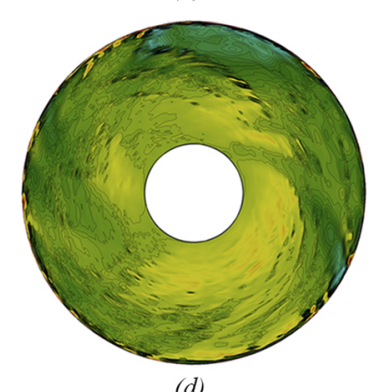

FIG. 16. A comparison of instantaneous distributions of the normalised volume fraction $\alpha$ contours for the $T_{P}$ model, at the rotor boundary layer, $R e_{\omega}=1 \times 10^{5}$, (a) $\phi=0.04$, (c) $\phi=0.2$; stator boundary layer, (b) $\phi=0.04$, (d) $\phi=0.2$. by assuming the homogeneous volume fractions, but the $T_{P}$ model considers nanoparticle slip velocities due to the thermophoresis and the Brownian forces. According to the $T_{P}$ simulation model, the thermophoresis forces act as a dominant force on the nanoparticles, which was driven away from the hot stator surfaces. In order to provide a quantitative comparison of the $S_{P}$ and the $T_{P}$ model predictions of heat transfer, we now look to obtain dimensionless heat transfer coefficients $N u_{r}$ in both cases.

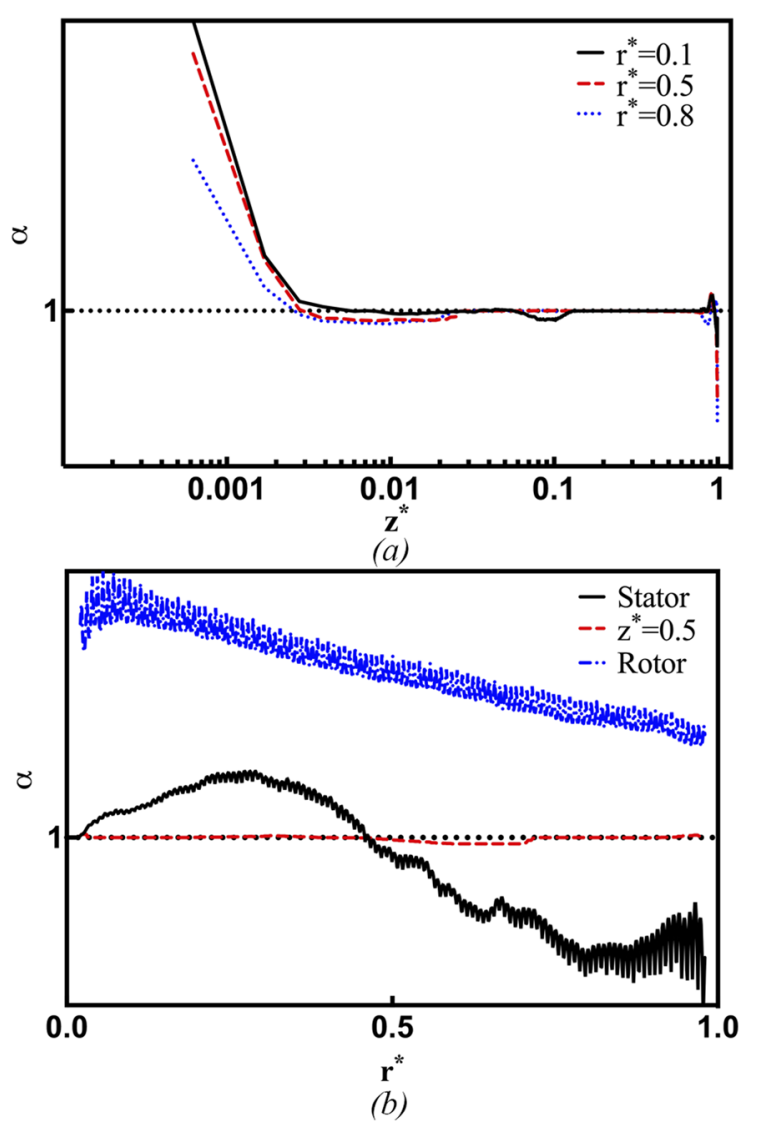

FIG. 17. The volume fraction distributions for $T_{P}$ model, $R e_{\omega}=1 \times 10^{5}$, (a) axial distributions at different radial positions, (b) radial distributions at the rotor, the mid-section, and the stator. 


\section{Nanofluid heat transfer coefficients in rotor-stator cavities}

The dimensionless Nusselt number is often used to quantify the efficiency of a heat-transfer process and we use it for that reason here. In particular, we use the local Nusselt number that is expressed as a function of the local radius of the stator disk.

The previous investigations of Nikitenko, ${ }^{9}$ Pellé and Harmand, ${ }^{67}$ and Tuliszka-Sznitko et al. ${ }^{3}$ studied the behavior of Nusselt numbers in various rotor-stator flow configurations. Each experienced a high dependency of local Nusselt numbers on local Reynolds numbers, which suggests that Nusselt numbers are sensitive to the local flow structures. In general, the local Nusselt number of a flow domain can be defined as

$$
N u_{r}=\frac{h_{T} r}{k_{T}} .
$$

Figure 18 shows the local Nusselt number distributions from both our $S_{P}$ and $T_{P}$ simulations. These are calculated at the stator boundary layer $\left(z^{*}=1\right)$, which is the heat source within our simulations. Consistent with the mean temperature distributions shown in Fig. 10, the local Nusselt numbers are found to increase as the volume fraction increases. All $S_{P}$ simulations are found to lead to marginally higher $N u_{r}$ than the respective $T_{P}$ simulation at all radial positions, which can be explained by considering the thermophoresis effects near the hot stator boundary layer. The local Nusselt numbers increase with the local radius of the disk (or $R e_{\omega, \text { local }}$ ), and most authors $3,9,67,68$ express the relationship as a power law,

$$
N u_{r}=a R e_{\omega, l o c a l}^{b} .
$$

Here $a$ and $b$ are multiplicative and exponential constants, respectively. The multiplicative constant depends on the temperature boundary conditions and the characteristic features of the rotor-stator cavity, and the exponential constant $b$ is a flow-dependent parameter. In most power law correlations, authors have used $b=0.5$ for the laminar flow and $b=0.8$ for the turbulent flow. The experimental investigation of Nikitenko ${ }^{9}$ predicts the values $a=0.0178$ and $b=0.8$, and the LES simulations of Tuliszka-Sznitko et $\mathrm{al}^{3}$ predict the values $a=0.021$ and $b=0.795$ for the turbulent stator of the rotor-stator cavities.

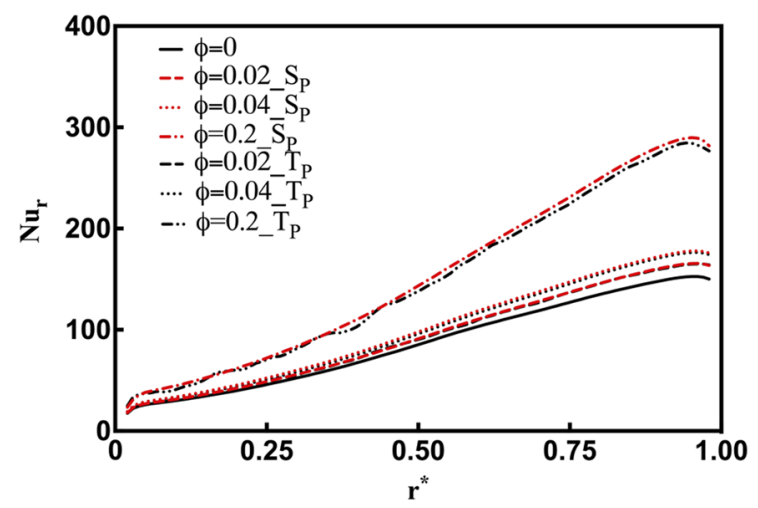

FIG. 18. A comparison of local Nusselt number distributions in the radial direction for the $S_{P}$ and the $T_{P}$ models at the stator boundary layer, $R e_{\omega}=1 \times 10^{5}$.
TABLE II. The fitted constant values of $a$ and $b$ for the model Eq. (23), RMSE values of the fitting, and the mean Nusselt numbers $\overline{\mathrm{Nu}}$.

\begin{tabular}{lcccr}
\hline \hline$\phi$ & $a$ & $b$ & RMSE & \multicolumn{1}{c}{$\overline{N u}$} \\
\hline 0.0 & 0.0158 & 0.807 & 3.88 & 87.19 \\
$0.02\left(S_{P}\right)$ & 0.0144 & 0.822 & 4.09 & 93.25 \\
$0.02\left(T_{P}\right)$ & 0.0142 & 0.823 & 4.00 & 92.92 \\
$0.04\left(S_{P}\right)$ & 0.0155 & 0.822 & 4.40 & 100.23 \\
$0.04\left(T_{P}\right)$ & 0.0132 & 0.834 & 4.30 & 98.54 \\
$0.2\left(S_{P}\right)$ & 0.0071 & 0.932 & 6.90 & 152.49 \\
$0.2\left(T_{P}\right)$ & 0.0063 & 0.940 & 6.60 & 148.57 \\
\hline \hline
\end{tabular}

Table II shows further analysis on the Nusselt number model in Eq. (23) and the Nusselt number distributions presented Fig. 18. We used MATLAB ${ }^{\circledR}$ "curve-fitting tool$b o x$ " to estimate the constants $a$ and $b$ in the power law Nusselt number model using our calculated Nusselt number distributions. The second and the third columns of Table II represent the estimated values of $a$ and $b$. In this case, the non-linear least square with the trusted region method ${ }^{69}$ is used to estimate the constants. The fourth column shows the respective values of the Root-Mean-Square Errors (RMSE) of the fits as calculated by

$$
\text { RMSE }=\sqrt{\frac{\sum\left(N u_{r, i}-\widehat{N u_{r, i}}\right)^{2}}{(n-m)}},
$$

where the $N u_{r, i}$ and the $\widehat{N u_{r, i}}$ are the $i$ th value to be estimated and the $i$ th estimated value, respectively. The constants $n$ and $m$ are the numbers of responses and the number of coefficients estimated from those responses. RMSE values close to zero are desirable and the values arising from the current models can be significantly yet artificially reduced by considering only the middle section of the Nusselt number distributions. However, here we use the entire distribution to estimate the model constants.

The adjusted $R^{2}=[0,1]$ value $e^{70,71}$ is a measure of the quality of the fit with values close to $R^{2}=1$ indicating a better fit. In all of our fitting models, $R^{2}$ is very close to $1\left(R^{2}>0.98\right)$. However, Spiess and Neumeyer ${ }^{72}$ have claimed that $R^{2}$ values alone are inadequate to estimate the quality of the non-linear fitting models. Hence we show in Fig. 19 a direct comparison between the Nusselt number distributions for the $S_{P}$ models with the nanoparticles volume fractions of $\phi=0.04$ and 0.2 and their corresponding fitted curves that were generated through the non-linear least square method.

According to the estimated model constants $a$ and $b$ in Table II, the (inceptive) nanoparticle volume fraction increases, and the multiplicative constant $a$ decreases (monotonically for the $T_{P}$ model), whereas the exponential parameter $b$ increases monotonically for both $S_{P}$ and $T_{P}$ models. We expected both model constants $a$ and $b$ to be increased at higher nanoparticle volume fractions. However, the reduction in the multiplicative constant, $a$, as the volume fraction increases cannot be explained as a direct consequence of the nanofluids. These higher multiplicative constants of lower nanoparticle volume fractions may result in higher heat transfer rates at the lower $R e_{\omega, \text { local }}$ when compared to the mixtures with 


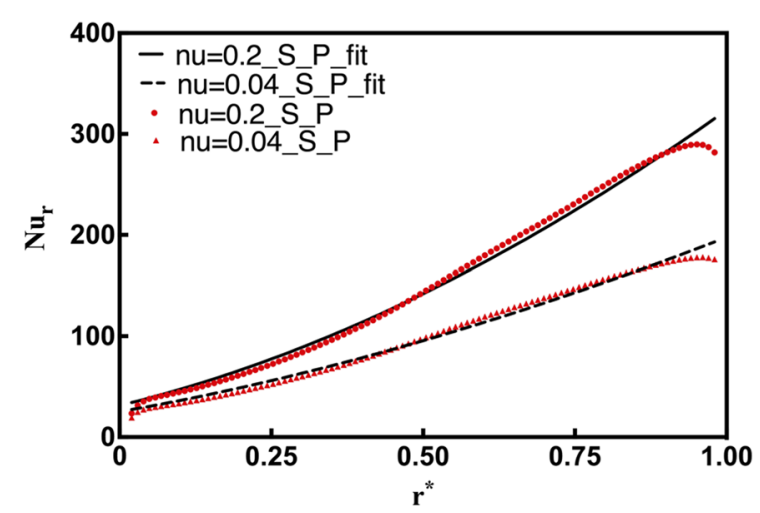

FIG. 19. A direct comparison between the local Nusselt number distributions for $\phi=0.2$ and 0.04 calculated from the $S_{P}$ model and the fitted curves are generated through the model constants $a$ and $b$, which were calculated using the non-linear least square method.

relatively higher nanoparticle volume fractions. For example, the $S_{P}$ model for pure water has higher heat transfer rates than the $S_{P}$ model of $\phi=0.2$ at $R e_{\omega, \text { local }}<1000$. A similar comparison of exponential constants suggests that the $T_{P}$ models have higher exponential constants, $b$, than their $S_{P}$ counterparts, and this could result in higher heat transfer rates of $T_{P}$ at higher $R e_{\omega}$,local, which is contrary to the results shown in Fig. 18. In the particular cases of the (inceptive) volume fraction of $\phi=0.2$ for $S_{P}$ and $T_{P}$ simulations, the crossover point of these two lines is expected to occur at $R e_{\omega, \text { local }} \sim 3 \times 10^{6}$. According to the arguments presented in Sec. III C, the nanoparticle displacement may be severe at higher $R e_{\omega}$, local, which results in a further decrease in heat transfer rates. Nevertheless, the calculated values for these model constants are admissible for the Reynolds numbers range of the current computational domain, $8 \times 10^{4}<R e_{\omega, \text { local }}<1 \times 10^{5}$. These observations suggest that the empirical power law Nusselt number model is not suitable for predicting generalised aspects related to rotor-stator cavities filled with nanofluids.

The fourth column of Table II represents the mean Nusselt numbers of the stator,

$$
\overline{N u}=\frac{\int_{r_{1}}^{r_{2}} N u_{r} 2 \pi r d r}{\pi\left(r_{2}^{2}-r_{1}^{2}\right)},
$$

calculated based on the local Nusselt number distributions. The $S_{P}$ and $T_{P}$ simulations with the nanoparticle (inceptive) volume fractions of $\phi=0.2$ show over $70 \%$ increase when compared to the base simulation of $\phi=0$. In this case, the mean Nusselt number of the $S_{P}$ simulation is about $4 \%$ higher than the similar $T_{P}$ simulation. This observation agrees with the previous explanation about the thermophoresis effects on the nanoparticles near a hot surface. However, the disparity between the $S_{P}$ and the $T_{P}$ simulations reduce as the nanoparticle volume fraction decreases.

\section{DISCUSSION AND CONCLUSIONS}

This study considered the development of two computational models to evaluate rotor-stator cavities filled with nanofluids. The first model is based on a single-phase transport model, in which the nanoparticle phase fraction is assumed to be constant throughout the simulation time. The second model is based on a two-phase transport model that assumes a slip velocity between the nanoparticles and the carrier phase; Brownian diffusion and thermophoresis effects are considered to be the plausible cause of these slip velocities. The two models have been used to simulate $\mathrm{Al}_{2} \mathrm{O}_{3}$ nanofluids with volume fractions $\phi=0.02,0.04,0.2$. In all simulations, the operational Reynolds number was fixed at $R e_{\omega}=1 \times 10^{5}$. The two models were validated using a pilot simulation with pure water as the working fluid (modeled by very small nanoparticle volume fraction of $\phi=2 \times 10^{-5}$ ).

The velocity fields obtained were found to be very similar in both the $S_{P}$ and $T_{P}$ model simulations. Mean radial, tangential, and axial velocity components of the rotor boundary layer were seen to increase with higher nanoparticle concentrations. Similar observations were made for the stator boundary layer, with the exception of the mean tangential velocity component which tends to decrease with increasing volume fractions. Both the radial and tangential turbulence intensity profiles were found to be reduced compared to pure water at a volume fraction $\phi=0.2$ but showed a slight increasing trend for volume fractions of $\phi=0.02$ and 0.04 . However, we have noted that the effects of nanoparticles on momentum transport are subtle and hence there would not be a significant improvement in convective heat transport solely because of nanoparticle volume fractions.

Nevertheless, instantaneous and mean temperature distributions are affected by the nanofluid, and they show elevated temperature distributions in the cases of higher nanoparticle volume fractions. This is primarily because of the higher thermal conductivities of nanofluids. However, careful comparisons between the simulation models have shown that the $S_{P}$ simulations lead to slightly elevated temperature distributions compared to their $T_{P}$ simulation counterparts. This can be explained by the capability of the $T_{P}$ model to capture the displacement of nanoparticles in the hot stator boundary, at which the thermophoresis forces are significant. Our work suggests that the thermophoresis force is the dominant particle force over the Brownian forces. Finally, the local Nusselt number and the mean Nusselt number distributions at the stator boundary have been obtained for all parameter and model cases and compared with a power law model in Eq. (23). The model was found to hold at all volume fractions despite it arising from an experimental study of conventional fluid. The mean Nusselt numbers show $6 \%-70 \%$ increase due to the different nanoparticle volume fractions of $\phi=0.02-0.2$. For highest nanoparticle volume fraction $\phi=0.2$, the mean Nusselt number of $S_{P}$ simulation is $4 \%$ higher than the similar $T_{P}$ simulation but the disparity is reduced as the nanoparticle volume fraction decreases.

Our simulations have been conducted for $\mathrm{Al}_{2} \mathrm{O}_{3}$ nanoparticles of a constant diameter of $30 \mathrm{~nm}$. Furthermore, numerical and experimental investigations can be conducted to extend the understanding of nanoparticles of different sizes and different types such as $\mathrm{Cu}, \mathrm{CuO}, \mathrm{TiO}_{2}, \mathrm{SiO}_{2}$, and $\mathrm{Ag}$, in the context of rotor-stator flows. This will potentially help to extend the conventional power law Nusselt number model to accommodate the characteristics and properties of the nanofluids. 


\section{ACKNOWLEDGMENTS}

This research used the ALICE High Performance Computing Facility at the University of Leicester, UK.

\section{NOMENCLATURE}

$\rho_{f} \quad$ Density of fluid

$\rho_{n f} \quad$ Density of the nanofluid

$\rho_{n p} \quad$ Density of nanoparticles

$P \quad$ Static pressure

$\alpha \quad$ Dimensionless volume fraction

$\beta \quad$ Thermal expansion coefficient

$\beta_{n f} \quad$ Thermal expansion coefficient of the nanofluid

$\mu_{f} \quad$ Dynamic viscosity of fluid

$\mu_{n f} \quad$ Dynamic viscosity of the nanofluid

$\mu_{s g s} \quad$ Subgrid-scale viscosity

$v \quad$ Kinematic viscosity of fluid

$\overline{\mathrm{Nu}} \quad$ Mean Nusselt number

$\Phi \quad$ Nanoparticle volume fraction

$\phi_{t_{0}} \quad$ Inceptive volume fraction/volume fraction at $t=0$

$\sqrt{R_{\theta \theta}}$

$\sqrt{R_{r r}}$

$B$

$c_{p}$

$D_{B}$

$D_{T}$

$G$

$g_{b}$

$h$

$h_{T}$

K

$k$

$k_{B}$

$k_{f}$

$k_{T}$

$k_{n f}$

$k_{n p}$

$k_{s g s}$

$\mathrm{Nu}_{r}$

$\mathrm{Pr}_{t}$

$P r_{s g s}$

$Q$

$r$

$r^{*}$

$r_{1}$

$r_{2}$

$R_{m}$

$r_{p}$

$R a$

$R e_{\omega, l o c a l}$

$R e_{\omega}$

$S_{P}$

$T$

$T_{P}$

$U$

$U_{\theta}$

$u_{\theta}$
$U_{\theta}^{*}$

$u_{r}$

$U_{r}^{*}$

$U_{r}^{*}$

$U_{z}^{*}$

$z$

$z^{*}$
Nondimensional mean resolved tangential velocity Instantaneous resolved radial velocity

Mean resolved radial velocity

Nondimensional mean resolved radial velocity

Nondimensional mean resolved axial velocity

Axial direction or axial distance

Dimensionless axial distance

${ }^{1}$ E. Serre, P. Bontoux, and B. Launder, "Transitional-turbulent flow with heat transfer in a closed rotor-stator cavity," J. Turbul. 5, 1-3 (2004).

${ }^{2}$ S. Poncet and E. Serre, "Large eddy simulation of non-isothermal turbulent rotor-stator flows," in The Twelfth International Symposium on Transport Phenomena and Dynamics of Rotating Machinery, 2008.

${ }^{3}$ E. Tuliszka-Sznitko, A. Zielinski, and W. Majchrowski, "LES of the nonisothermal transitional flow in rotating cavity," Int. J. Heat Fluid Flow 30, 534-548 (2009).

${ }^{4}$ E. Tuliszka-Sznitko and W. Majchrowski, "LES and DNS of the flow with heat transfer in rotating cavity," Comput. Methods Sci. Technol. 16, 105114 (2010).

${ }^{5}$ E. Tuliszka-Sznitko, W. Majchrowski, and K. Kiełczewski, "Investigation of transitional and turbulent heat and momentum transport in a rotating cavity,” Int. J. Heat Fluid Flow 35, 52-60 (2012).

${ }^{6}$ T. V. Kármán, "Über laminare und turbulente reibung," ZAMM- J. Appl. Math. Mech./Z. Angew. Math. Mech. 1, 233-252 (1921).

${ }^{7} \mathrm{~K}$. Millsaps, "Heat transfer by laminar flow from a rotating plate," J. Aeronaut. Sci. 18, 354-355 (1951).

${ }^{8}$ E. Cobb and O. Saunders, "Heat transfer from a rotating disk," Proc. R. Soc. A 236, 343-351 (1956).

${ }^{9} \mathrm{~N}$. Nikitenko, "Experimental investigation of heat exchange of a disk and a screen," J. Eng. Phys. 6, 1-11 (1963).

${ }^{10}$ J. Owen, C. Haynes, and F. Bayley, "Heat transfer from an air-cooled rotating disk," Proc. R. Soc. A 336, 453-473 (1974).

${ }^{11}$ J. W. Daily and R. E. Nece, "Chamber dimension effects on induced flow and frictional resistance of enclosed rotating disks," J. Basic Eng. 82, 217-230 (1960).

${ }^{12}$ J. M. Owen and R. H. Rogers, Flow and Heat Transfer in Rotating-Disc Systems (Research Studies Press, 1989).

${ }^{13} \mathrm{~J}$. Owen and R. H. Rogers, Flow and Heat Transfer in Rotating Disc Systems, Volume 2: Rotating Cavities (Research Studies Press, 1995).

${ }^{14}$ A. J. Faller, "Instability and transition of disturbed flow over a rotating disk," J. Fluid Mech. 230, 245-269 (1991).

${ }^{15}$ M. Itoh, Y. Yamada, S. Imao, and M. Gonda, "Experiments on turbulent flow due to an enclosed rotating disk," Exp. Therm. Fluid Sci. 5, 359-368 (1992).

${ }^{16}$ H. S. Littell and J. K. Eaton, "Turbulence characteristics of the boundary layer on a rotating disk," J. Fluid Mech. 266, 175-207 (1994).

${ }^{17}$ R. Lingwood, "An experimental study of absolute instability of the rotatingdisk boundary-layer flow," J. Fluid Mech. 314, 373-405 (1996).

${ }^{18}$ R. J. Lingwood, "Absolute instability of the Ekman layer and related rotating flows," J. Fluid Mech. 331, 405-428 (1997).

${ }^{19}$ G. Gauthier, P. Gondret, and M. Rabaud, "Axisymmetric propagating vortices in the flow between a stationary and a rotating disk enclosed by a cylinder,” J. Fluid Mech. 386, 105-126 (1999).

${ }^{20} \mathrm{C}$. J. Elkins and J. K. Eaton, "Turbulent heat and momentum transport on a rotating disk," J. Fluid Mech. 402, 225-253 (2000).

${ }^{21}$ C. Subramanian and R. Antonia, "Effect of Reynolds number on a slightly heated turbulent boundary layer," Int. J. Heat Mass Transfer 24, 1833-1846 (1981).

${ }^{22}$ M. Gibson, C. Verriopoulos, and N. Vlachos, "Turbulent boundary layer on a mildly curved convex surface," Exp. Fluids 2, 17-24 (1984).

${ }^{23}$ J. M. Wallace, "Quadrant analysis in turbulence research: History and evolution," Annu. Rev. Fluid Mech. 48, 131-158 (2016).

${ }^{24}$ M. Turkyilmazoglu, "Flow and heat simultaneously induced by two stretchable rotating disks," Phys. Fluids 28, 043601 (2016).

${ }^{25}$ J. C. Maxwell, A Treatise on Electricity and Magnetism (Clarendon Press, 1881), Vol. 1.

${ }^{26}$ S. K. Das, S. U. Choi, and H. E. Patel, "Heat transfer in nanofluids-A review," Heat Transfer Eng. 27, 3-19 (2006).

${ }^{27}$ S. U. Choi and J. A. Eastman, "Enhancing thermal conductivity of fluids with nanoparticles," Technical Report ANL/MSD/CP-84938; CONF-95113529, Argonne National Laboratory, IL, USA, 1995. 
${ }^{28}$ K. Khanafer, K. Vafai, and M. Lightstone, "Buoyancy-driven heat transfer enhancement in a two-dimensional enclosure utilizing nanofluids," Int. J. Heat Mass Transfer 46, 3639-3653 (2003).

${ }^{29}$ Y. Xuan and Q. Li, "Investigation on convective heat transfer and flow features of nanofluids," J. Heat Transfer 125, 151-155 (2003).

${ }^{30}$ S. Z. Heris, M. N. Esfahany, and S. G. Etemad, "Experimental investigation of convective heat transfer of $\mathrm{Al}_{2} \mathrm{O}_{3}$ /water nanofluid in circular tube," Int. J. Heat Fluid Flow 28, 203-210 (2007).

${ }^{31}$ C. T. Nguyen, G. Roy, C. Gauthier, and N. Galanis, "Heat transfer enhancement using $\mathrm{Al}_{2} \mathrm{O}_{3}$-water nanofluid for an electronic liquid cooling system," Appl. Therm. Eng. 27, 1501-1506 (2007).

${ }^{32}$ M. Alinia, D. Ganji, and M. Gorji-Bandpy, "Numerical study of mixed convection in an inclined two sided lid driven cavity filled with nanofluid using two-phase mixture model," Int. Commun. Heat Mass Transfer 38, 1428-1435 (2011).

${ }^{33}$ M. Goodarzi, M. Safaei, K. Vafai, G. Ahmadi, M. Dahari, S. Kazi, and N. Jomhari, "Investigation of nanofluid mixed convection in a shallow cavity using a two-phase mixture model," Int. J. Therm. Sci. 75, 204-220 (2014).

${ }^{34} \mathrm{~B}$. Ghasemi and S. Aminossadati, "Brownian motion of nanoparticles in a triangular enclosure with natural convection," Int. J. Therm. Sci. 49, 931940 (2010).

${ }^{35}$ J. Koo and C. Kleinstreuer, "Laminar nanofluid flow in microheat-sinks," Int. J. Heat Mass Transfer 48, 2652-2661 (2005).

${ }^{36}$ J. Buongiorno, "Convective transport in nanofluids," J. Heat Transfer 128, 240-250 (2006).

${ }^{37}$ A. Mahajan and M. K. Sharma, "Penetrative convection in magnetic nanofluids via internal heating," Phys. Fluids 29, 034101 (2017).

${ }^{38}$ A. Avramenko, I. Shevchuk, S. Abdallah, D. Blinov, and A. Tyrinov, "Selfsimilar analysis of fluid flow, heat, and mass transfer at orthogonal nanofluid impingement onto a flat surface," Phys. Fluids 29, 052005 (2017).

${ }^{39}$ T. Hayat, T. Muhammad, A. Alsaedi, and B. Ahmad, "Three-dimensional flow of nanofluid with Cattaneo-Christov double diffusion," Results Phys. 6, 897-903 (2016).

${ }^{40}$ M. Ramzan, M. Bilal, J. D. Chung, D. C. Lu, and U. Farooq, "Impact of generalized Fourier's and Fick's laws on MHD 3D second grade nanofluid flow with variable thermal conductivity and convective heat and mass conditions," Phys. Fluids 29, 093102 (2017).

${ }^{41}$ N. Bachok, A. Ishak, and I. Pop, "Flow and heat transfer over a rotating porous disk in a nanofluid,” Phys. B 406, 1767-1772 (2011).

${ }^{42}$ H. E. Patel, K. Anoop, T. Sundararajan, and S. K. Das, "A micro-convection model for thermal conductivity of nanofluids," in International Heat Transfer Conference (Begel House, Inc., 2006), p. 13.

${ }^{43}$ M. Turkyilmazoglu, "Nanofluid flow and heat transfer due to a rotating disk," Comput. Fluids 94, 139-146 (2014).

${ }^{44}$ M. Mustafa, J. A. Khan, T. Hayat, and A. Alsaedi, "On Bödewadt flow and heat transfer of nanofluids over a stretching stationary disk," J. Mol. Liq. 211, 119-125 (2015).

${ }^{45}$ A. Mushtaq and M. Mustafa, "Computations for nanofluid flow near a stretchable rotating disk with axial magnetic field and convective conditions," Results Phys. 7, 3137-3144 (2017).

${ }^{46}$ É. Séverac, S. Poncet, É. Serre, and M.-P. Chauve, "Large eddy simulation and measurements of turbulent enclosed rotor-stator flows," Phys. Fluids 19, 085-113 (2007).

${ }^{47}$ S. Makino, M. Inagaki, and M. Nakagawa, "Laminar-turbulence transition over the rotor disk in an enclosed rotor-stator cavity," Flow, Turbul. Combust. 95, 399-413 (2015).

${ }^{48}$ V. Ekman, "On the influence of the earth's rotation on ocean-currents," Ark. Mat., Astron. Fys. 2, 1-53 (1905).

${ }^{49}$ U. T. Bödewadt, "Die drehströmung über festem grunde," ZAMM- Z. Angew. Math. Mech. 20, 241-253 (1940).
${ }^{50}$ J. Tu, G. H. Yeoh, and C. Liu, Computational Fluid Dynamics: A Practical Approach (Butterworth-Heinemann, 2012).

${ }^{51}$ F. Moukalled, L. Mangani, M. Darwish et al., The Finite Volume Method in Computational Fluid Dynamics (Springer, 2016).

${ }^{52}$ R. I. Issa, A. Gosman, and A. Watkins, "The computation of compressible and incompressible recirculating flows by a non-iterative implicit scheme," J. Comput. Phys. 62, 66-82 (1986).

${ }^{53}$ F. Nicoud and F. Ducros, "Subgrid-scale stress modelling based on the square of the velocity gradient tensor," Flow, Turbul. Combust. 62, 183-200 (1999).

${ }^{54}$ M. Antonopoulos-Domis, "Large-eddy simulation of a passive scalar in isotropic turbulence," J. Fluid Mech. 104, 55-79 (1981).

${ }^{55}$ D. D. Gray and A. Giorgini, "The validity of the Boussinesq approximation for liquids and gases," Int. J. Heat Mass Transfer 19, 545-551 (1976).

${ }^{56}$ M. Manninen, V. Taivassalo, S. Kallio et al., On the Mixture Model for Multiphase Flow (VTT Publications, 1996).

${ }^{57}$ M. Rebay, S. Kakaç, and R. M. Cotta, Microscale and Nanoscale Heat Transfer: Analysis, Design, and Application (CRC Press, 2016).

${ }^{58}$ G. McNab and A. Meisen, "Thermophoresis in liquids," J. Colloid Interface Sci. 44, 339-346 (1973).

${ }^{59}$ A. Einstein, "Eine neue bestimmung der moleküldimensionen," Ann. Phys. 324, 289-306 (1906).

${ }^{60}$ H. Brinkman, "The viscosity of concentrated suspensions and solutions," J. Chem. Phys. 20, 571 (1952).

${ }^{61}$ N. A. A. Bakar, N. Bachok, and N. M. Arifin, "Nanofluid flow using Buongiorno model over a stretching sheet and thermophysical properties of nanoliquids," Indian J. Sci. Technol. 9, 1-9 (2016).

${ }^{62}$ T. Bridel-Bertomeu, L. Gicquel, and G. Staffelbach, "Large scale motions of multiple limit-cycle high Reynolds number annular and toroidal rotor/stator cavities," Phys. Fluids 29, 065115 (2017).

${ }^{63}$ D. Fernando, S. Gao, and S. Garrett, "The effect of surface roughness on rotor-stator cavity flows," Phys. Fluids 30, 064103 (2018).

${ }^{64}$ J. Jeong and F. Hussain, "On the identification of a vortex," J. Fluid Mech. 285, 69-94 (1995).

${ }^{65}$ C. Hu, P. Heng, M. Bai, J. Lv, Y. Wang, and X. Li, "Numerical study of nanofluids flow characteristics using LES-Lagrange method and molecular dynamics simulation," in ASME 2013 4th International Conference on Micro/Nanoscale Heat and Mass Transfer (American Society of Mechanical Engineers, 2013), p. V001T02A001.

${ }^{66}$ O. Ghaffari, A. Behzadmehr, and H. Ajam, "Turbulent mixed convection of a nanofluid in a horizontal curved tube using a two-phase approach," Int. Commun. Heat Mass Transfer 37, 1551-1558 (2010).

${ }^{67}$ J. Pellé and S. Harmand, "Heat transfer measurements in an opened rotor-stator system air-gap," Exp. Therm. Fluid Sci. 31, 165-180 (2007).

${ }^{68}$ L. Dorfman, Hydrodynamic Resistance and Heat Loss of Rotating Solids (Oliver and Boyd, Edinburgh and London, 1963).

${ }^{69} \mathrm{C}$. $\mathrm{Xu}$, "Nonlinear least squares: Trust region methods nonlinear least squares: Trust region methods," in Encyclopedia of Optimization, edited by C. A. Floudas and P. M. Pardalos (Springer US, Boston, MA, 2009), pp. 2630-2637.

${ }^{70}$ A. C. Cameron and F. A. Windmeijer, "An R-squared measure of goodness of fit for some common nonlinear regression models, ' J. Econom. 77, 329_ 342 (1997).

${ }^{71}$ M. Jeremy, "R squared, adjusted R squared," in Encyclopedia of Statistics in Behavioral Science (American Cancer Society, 2005).

${ }^{72}$ A.-N. Spiess and N. Neumeyer, "An evaluation of R2 as an inadequate measure for nonlinear models in pharmacological and biochemical research: A Monte Carlo approach," BMC Pharmacol. 10, 6 (2010). 